\title{
Wie können „bildungsferne“ Gruppen für ein Hochschulstudium gewonnen werden?
}

\author{
Eine empirische Simulation mit Implikationen für die \\ Steuerung des Bildungswesens
}

\section{Rolf Becker}

Zusammenfassung: Ausgehend davon, dass es in Deutschland bei einer relativ niedrigen Studienanfänger- und Akademikerquote eine ausgeprägte soziale Ungleichheit beim Hochschulzugang gibt, werden neben den Strukturen des Bildungssystems primäre und sekundäre Herkunftseffekte als Gründe dafür in Betracht gezogen, dass „,bildungsferne“ Gruppen an deutschen Hochschulen unterrepräsentiert sind. Empirischen Befunden für Abiturienten zufolge sind eher sekundäre Herkunftseffekte als primäre Herkunftseffekte ausschlaggebend dafür, ein Studium erfolgreich bewältigen zu können. Neutralisiert man bei Kontrolle der individuellen Erfolgserwartungen die sekundären Herkunftseffekte, so kann die Studienbeteiligung von Arbeiterkindern sehr viel deutlicher gesteigert werden als über die Neutralisierung der primären Herkunftseffekte. Neutralisiert man zudem die sekundären Herkunftseffekte bei den Übergängen in die Sekundarstufe I und II, so können die Hochschulzugänge „,bildungsferner“ Gruppen noch weiter gesteigert werden. Insgesamt sind sowohl die Selektions- und Filterprozesse des deutschen Schulsystems als auch die darauf basierenden Bildungsentscheidungen kontraproduktiv, wenn es darum geht, „bildungsferne" Gruppen für ein Hochschulstudium zu gewinnen.

Schlüsselwörter: Höhere Bildung · Hochschulzugang · Primäre und sekundäre Herkunftseffekte $\cdot$ Soziale Ungleichheit · Bildungschancen · Ausbildungsentscheidung

\section{What Could be Done in Order to Motivate the Working Classes for University Education? An Empirical Simulation with Implications for Educational Policies}

\begin{abstract}
Against the background of the relatively low enrolment of students at universities, the minor proportion of academics at the population, and the distinctive social inequality at the transition to the tertiary education in Germany, it is assumed that - additionally to the structure
\end{abstract}

\footnotetext{
(C) VS-Verlag 2009

R. Becker $(\bowtie)$

Institut für Erziehungswissenschaft, Abteilung Bildungssoziologie

Universität Bern, Muesmattstrasse 27, 3012 Bern, Schweiz

E-Mail: rolf.becker@edu.unibe.ch
} 
of the educational system - primary and secondary effects of social origin are responsible for that the working class children are underrepresented at German universities. According to empirical findings for high-school graduates, their subjectively expected success in university training in general as well as their educational decision on continuing tertiary education, in particular, are crucial for this empirical fact of persistent inequalities of university education. If one cancels out the secondary effects of social origin, the participation of working class children could be increased more significantly than by neutralizing the primary effects. If one neutralizes also the secondary effects at both, the first and the second transition in the individuals' educational course, the individuals' transition to the sector of tertiary education could be increased additionally. Both, the selection and filter processes of the Germany school system as well as individuals' educational choices provide the counterproductive effect that the talented offspring from socially disadvantaged classes will be diverted away from the higher education and the universities.

Keywords: Higher education - Tertiary training - Primary and secondary effects · Social inequality of educational opportunities $\cdot$ Educational decision

\section{Einleitung}

Die soziale Frage des Hochschulzugangs ist wieder zu einem der wichtigsten Probleme für die Hochschulentwicklung in Deutschland geworden (Dobischat 2007). Während in den 1960er Jahren vornehmlich der ökonomische Bedarf an Akademikern, der durch bislang unerschlossene Bildungsreserven gedeckt werden sollte, die hochschulpolitische Debatte bestimmte (Picht 1964), standen von Mitte der 1960er bis Anfang der 1970er Jahre eher gesellschaftspolitische Forderungen nach höherer Bildung als Bürgerrecht im Vordergrund (Dahrendorf 1976). Denn höhere Bildung wurde bis dahin vornehmlich den Mädchen sowie den Angehörigen der Arbeiterschichten vorenthalten (Dahrendorf 1965a, 1965b; Pross 1969). Nach kontrovers geführten Debatten in den 1980er Jahren über eine vermeintliche „Akademikerschwemme“ bei einem gleichzeitigen Mangel an Lehrlingen und qualifizierten Absolventen beruflicher Ausbildungen (Lüttinger 1994) wird seit Mitte der 1990er Jahre unter den Vorzeichen von „brain drain“ wiederum ein Mangel an Akademikern und Fachhochschulabsolventen beklagt (Mayer 2003, S. 585).

Auch die OECD bemängelt in den letzten Jahren regelmäßig in ihrem Bericht „Education At A Glance“ die im Vergleich zum OECD-Durchschnitt (36 Prozent im Jahre 2007) geringe Akademikerquote in Deutschland (20 Prozent). ${ }^{1}$ Ebenso wird die ausgeprägte Abhängigkeit des Bildungserfolgs von der sozialen Herkunft moniert. Demnach studieren deutlich weniger Arbeiterkinder an Deutschlands Universitäten und Fachhochschulen als Kinder von Akademikern (Müller u. Pollak 2008; Mayer et al. 2007). ${ }^{2}$ Ähnlich wie in den

1 In Deutschland stieg die Studierendenquote (prozentualer Anteil der Studierenden an der Gesamtbevölkerung im Alter zwischen 20 und 30 Jahren) von 5 Prozent im Jahre 1970 auf über 8 Prozent im Jahre 1980 und schließlich auf rund 10 Prozent im Jahre 1990 und nach der deutschen Einheit auf 14 Prozent im Jahre 2003. Dass die von der OECD vorgelegten Statistiken erhebliche methodische Mängel aufweisen und zu irreführenden Ergebnissen beim internationalen Vergleich führen, ist an dieser Stelle ohne Belang.

2 Die Studienanfängerquote für das Studium an Universitäten (einschließlich pädagogischer und theologischer Hochschulen) und Fachhochschulen (relativer Anteil der Studienanfänger an der 18- bis unter 22-jährigen Bevölkerung) ist von 19 Prozent im Jahre 1980 auf rund 31 Prozent 
1960er und 1970er Jahren wird nunmehr gefordert, dass der Hochschulzugang erheblich erleichtert werden müsse, um die aufgrund der demographischen Entwicklung ohnehin knappen Bildungsreserven in Deutschland auszuschöpfen (OECD 2007). Diese Reserven werden vor allem bei Kindern aus „,bildungsfernen“ Schichten gesehen. Daraus ergibt sich folgende empirisch zu untersuchende Frage: „Wie können ,bildungsferne“ Gruppen für ein Hochschulstudium gewonnen werden?“" (Mayer 2003, S. 608).

Im zweiten Abschnitt werden auf Grundlage eines theoretischen Modells zwei Ursachenkomplexe für die soziale Ungleichheit des Hochschulzugangs diskutiert. Anhand von im dritten Abschnitt vorgestellten Daten über die Ausbildungsentscheidung von Abiturienten sowie die elterliche Bildungsentscheidung beim Übergang in die Sekundarstufe I werden im vierten Abschnitt die Gewichte primärer und sekundärer Herkunftseffekte isoliert und die Folgen potenzieller Maßnahmen, um diese Herkunftseffekte zu reduzieren oder gänzlich zu neutralisieren, simuliert. Eine Zusammenfassung der Befunde und Diskussion bildungspolitischer Implikationen erfolgt im fünften Abschnitt.

\section{Theoretische Grundlagen}

Um Talente aus „,bildungsfernen“ Gruppen wie etwa Arbeiterkinder an die Hochschulen zu bringen, müssen die Ursachen für ihre Studienabstinenz bekannt sein, um potenziell wirksame Maßnahmen ableiten zu können. Als eine der gewichtigen Ursachen für dauerhafte soziale Ungleichheit beim Hochschulzugang gelten institutionelle Strukturen und Regelungen des Bildungssystems, welche die Bildungsentscheidungen von Individuen und Familien in der Art und Weise kanalisieren, dass vor allem „bildungsferne“ Gruppen vom Weg zur Universität oder Fachhochschule abgelenkt werden (Müller u. Pollak 2008; Becker u. Hecken 2008). In Deutschland ist die gymnasiale Oberstufe der „Flaschenhals“ und schließlich der Erwerb der Hochschulberechtigung ein „Nadelöhr“ auf dem Weg zur höheren Bildung (Mayer et al. 2007). Aber bereits auf dem Weg zum Gymnasium, zum Abitur und dann zur Hochschule sind an den einzelnen Übergangsstellen systematische Ablenkungswirkungen des stratifizierten, segmentierten und wenig durchlässigen Bildungssystem offensichtlich, die gerade Arbeiterkinder am Zugang zur tertiären Ausbildung ,hindern“. Selbst nach Erwerb der Hochschulreife wechseln Arbeiterkinder

bis Ende der 1980er Jahre gestiegen. Nach 1990 ist ein Anstieg der Studienanfängerquote von 29 auf 37 Prozent im Jahre 2003 zu verzeichnen. Zwar stieg bei Berücksichtigung des Studienbeginns an Universitäten und Fachhochschulen für Arbeiterkinder in der Zeit von 1982 bis 2000 die Studienanfängerquote von 9 auf 12 Prozent, aber im gleichen Zeitraum nahm die Studienanfängerquote bei den ohnehin sozial privilegierten Beamtenkindern von 46 auf 72 Prozent zu. Da im Jahre 1982 Beamtenkinder eine 9-mal bessere Chance als Arbeiterkinder hatten, an Universitäten oder Fachhochschulen zu studieren, und dieses Chancenverhältnis auf das 19-fache im Jahre 2000 gestiegen ist, ist die Bildungsexpansion beim Hochschulzugang mit einer deutlichen Anhebung sozial ungleicher Studienchancen einhergegangen. Gemessen an den gestiegenen Studienchancen von sozial privilegierten Personen aus höheren Sozialschichten, haben sich die relativ ungünstigen Studienchancen von Arbeiterkindern seit Anfang der 1980er Jahre kaum verändert. Auch der Ausbau der Fachhochschulen hat für die Arbeiterkinder allenfalls in einem geringfügigen Ausmaß zu besseren Studienchancen geführt (Becker 2006). 
eher in das duale Berufsbildungssystem als in die tertiäre Bildungslaufbahn. Somit erfolgt die Ablenkung der Arbeiterkinder vom Weg zur Hochschule durch selektive Anreize und Restriktionen, die mit der Opportunitätsstruktur des deutschen Bildungssystems verbunden sind (Müller u. Pollak 2008).

Um die Ablenkung studienbegabter Arbeiterkinder vom Hochschulstudium beeinflussen zu können, müssen wiederum die Ursachen dafür bekannt sein: Warum werden Arbeiterkinder im hochgradig stratifizierten, segmentierten und viele Bildungsentscheidungen abverlangenden Bildungssystem vom Hochschulstudium abgelenkt? Welche Push- und Pull-Faktoren sind hierfür ausschlaggebend? Wie wirken die mit der Opportunitätsstruktur des deutschen Bildungssystems verbundenen Anreize und Restriktionen? Eine theoriegeleitete und inzwischen empirisch fundierte Antwort auf die Frage lautet: Die Ablenkung der Arbeiterkinder vom Hochschulstudium und ihre Unterrepräsentation an Deutschlands Hochschulen sind aggregierte Konsequenzen, die sich (1) aus kumulativen individuellen Bildungsentscheidungen in Abhängigkeit von der sozialen Positionierung des Elternhauses im Schichtgefüge und dem Angebot von Bildungsgelegenheiten sowie (2) aus individuellen, mit sozialer Herkunft korrelierender Schulleistungen und Erfolgserwartungen in Abhängigkeit leistungsbezogener Selektion durch das Bildungssystem ergeben (Becker u. Hecken 2008).

\subsection{Primäre und sekundäre Effekte der sozialen Ungleichheit}

Zwischen den Sozialschichten systematisch variierende Push- und Pull-Faktoren lassen sich anhand der von Boudon (1974) vorgeschlagenen Unterscheidung von primären und sekundären Herkunftseffekten spezifizieren (Erikson u. Jonsson 1996; Goldthorpe 1996; Breen u. Goldthorpe 1997). Zum einen erlangen Kinder aus höheren Sozialschichten infolge der Ausstattung des Elternhauses sowie der Sozialisation und gezielten Förderung im anregungsreichen Elternhaus eher Fähigkeiten und Motivationen, die in der Schule und Ausbildung vorteilhaft sind. Aufgrund dieser günstigen ökonomischen, kulturellen und sozialen Voraussetzungen im Elternhaus weisen Kinder aus höheren Sozialschichten eher bessere Schulleistungen und höhere Erfolgserwartungen auf, während Arbeiterkinder aufgrund ihrer sozialen Herkunft oftmals kognitive Nachteile haben und ihren Bildungsverlauf in der Regel mit ungünstigen Lernvoraussetzungen beginnen. Da Kinder aus höheren Sozialschichten oftmals besser den jeweiligen schulischen Leistungsanforderungen gerecht werden, haben sie vergleichsweise größere Chancen, auf das Gymnasium wechseln und mit dem Abitur die Berechtigung für ein Studium erwerben zu können (primärer Effekt der sozialen Herkunft). Im Vergleich zu den Arbeiterkindern weisen sie auch höhere Erwartungen, ein Hochschulstudium erfolgreich bewältigen zu können, und ein geringeres Risiko auf, an leistungsbezogenen Hürden wie etwa dem Numerus Clausus zu scheitern. ${ }^{3}$

3 So konnten Becker u. Hecken (2008) für sächsische Abiturienten und Trautwein et al. (2006) für Abiturienten in Baden-Württemberg empirisch nachweisen, dass die sozialen Disparitäten des erwarteten Studienerfolgs mit verantwortlich dafür sind, dass sich Arbeiterkinder statt für ein Studium eher für eine Berufsausbildung entscheiden. Arbeiterkinder erwarten im Unterschied $\mathrm{zu}$ Abiturienten aus den Mittel- und Oberschichten in einem geringeren Maße, ein Studium erfolgreich abschließen zu können. 
Zum anderen hängt der weitere Bildungsweg in der Schule bis hin zu den Hochschulen zunächst von elterlichen und im späteren Bildungsverlauf maßgeblich von individuellen Bildungsentscheidungen der noch im Bildungssystem verbliebenen Jugendlichen ab. Dies ist vor allem nach Abschluss der Pflichtschulzeit der Fall, wenn sich Schulabgänger für eine Berufsausbildung oder für eine fortgesetzte Schulausbildung bis hin zum Hochschulstudium entscheiden müssen (Becker u. Hecken 2007; Hillmert u. Jacob 2003). Diese Entscheidungen variieren in Abhängigkeit von ökonomischen Ressourcen des Elternhauses sowie dessen sozialer Distanz zum System höherer Bildung deutlich zwischen den Sozialschichten (Boudon 1974). Somit unterscheiden sich Kinder verschiedener sozialer Herkunft selbst bei gleichen Leistungen oder bei gleicher Leistungsfähigkeit darin, ob sie die schulische Ausbildung auf einer gegebenen Bildungsstufe beenden oder sie mit einer tertiären Bildung fortsetzen (sekundärer Effekt der sozialen Herkunft). ${ }^{4}$ Die sozioökonomische Ausgangsverteilung bei den Studienberechtigten trägt bei gegebenen Leistungsfähigkeiten und Erfolgserwartungen über sozial selektive Ausbildungsentscheidungen zu Ungleichheiten beim Hochschulzugang nach sozialer Herkunft bei. ${ }^{5}$

In Anlehnung an Erikson u. Jonsson (1996) können diese theoretischen und inzwischen mehrfach empirisch bewährten Argumente für die schichtspezifische Wahl zwischen einer nichttertiären Berufsausbildung und dem Hochschulstudium in folgender Weise rekonstruiert werden (vgl. Stockè 2007; Becker 2000): Aus einer Auswahl möglicher Bildungswege entscheiden sich Abiturienten für diejenige Alternative, die bei einer Abwägung von erwarteten Erträgen und Kosten sowie dem wahrscheinlichen Bildungserfolg den größten Nutzen erbringen. Für die einzelnen Bildungsalternativen bilden die Abiturienten Erwartungswerte für den Nutzen EU (expected utility), die sie miteinander vergleichen. Diese Erwartungswerte ergeben sich aus dem subjektiv eingeschätzten Wert für den Ertrag der jeweiligen Ausbildungsabschlüsse B (benefit), dem Wert für die erwarteten direkten und indirekten Kosten C (cost) für die einzelnen Ausbildungsalternativen und dem Wert für die Wahrscheinlichkeit p (probability), die avisierte Ausbildung erfolgreich abschließen zu können. Diesen Zusammenhang formalisieren Erikson u. Jonsson (1996, S. 14) in folgender Weise: EU(.)=(B-C)p-C(1-p). Eine vereinfachte Gleichung ergibt schließlich: $\mathrm{EU}()=.\mathrm{p} \cdot \mathrm{B}-\mathrm{C}$. Die Entscheidung für eine nichttertiäre berufliche Ausbildung A oder für ein Hochschulstudium S erfolgt demnach in Abhängigkeit des jeweils erwarteten Nutzens EU(.) einer Ausbildungsalternative. Ein Abiturient entscheidet sich

4 Diese Logik gilt nicht nur für die Ausbildungsentscheidungen, sondern auch für die elterlichen Bildungsentscheidungen am Ende der Grundschulzeit ihrer Kinder (vgl. Stockè 2007; Becker 2000).

5 Für sächsische Abiturienten stellen Becker u. Hecken (2007) fest, dass Arbeiterkinder aufgrund vergleichsweise niedrigerer Bildungsmotivation, die sich aus den subjektiv erwarteten Bildungsrenditen und dem Statuserhaltmotiv ergibt, eher auf ein Universitätsstudium verzichten als Angehörige höherer Sozialschichten. Des Weiteren bringt ein hohes Investitionsrisiko, das Verhältnis von erwarteten Studienkosten und der Wahrscheinlichkeit, ein Studium erfolgreich bewältigen zu können, die Arbeiterkinder eher dazu, auf ein Studium zu verzichten, als dies bei Absolventen aus höheren Sozialschichten der Fall ist (Becker u. Hecken 2007, S. 112, 2009, S. 38). Offensichtlich sind entsprechend der tatsächlichen Verteilung von Finanzkapital zwischen den Sozialschichten subjektive Einschätzungen der mit dem Studium verbundenen Kosten mitentscheidend für einen Verzicht auf ein Hochschulstudium. 
demnach höchstwahrscheinlich für ein Hochschulstudium, wenn gilt: $E U(S)=p B(S)-$ $\mathrm{C}(\mathrm{S})>\mathrm{EU}(\mathrm{A})=\mathrm{pB}(\mathrm{A})-\mathrm{C}(\mathrm{A})$. Bei einem erwarteten Misserfolg erscheint der Bildungsertrag einer Ausbildungsalternative gleich Null, während die Kosten den Wert C beibehalten und den subjektiv erwarteten Nutzen einer Ausbildung bestimmen.

Da bei für alle Sozialschichten identischen Bildungserträgen die Erfolgserwartungen bei den Arbeiterkindern vergleichsweise geringer sind, werden sie aufgrund der überproportional höheren Kosten, die bei einem Hochschulstudium anfallen, eher eine nichttertiäre Berufsausbildung wählen. Weil zudem bei gegebenen Erfolgserwartungen den höheren Sozialschichten die gesamten Ausbildungskosten geringer als der Arbeiterschicht erscheinen, werden sie sich eher für länger andauernde Ausbildung wie dem Hochschulstudium entscheiden können. Im Aggregat ergeben diese individuellen, zwischen den sozialen Gruppen differierenden Ausbildungsentscheidungen die zu erklärenden Phänomene wie etwa schichtspezifische Studienanfängerquoten oder „Bildungsferne“ der Arbeiterkinder.

\subsection{Zwischenfazit}

Aus den vorherigen Ausführungen ist für Deutschland folgendes Zwischenfazit zu ziehen: Das Zusammenspiel von primären und sekundären Herkunftseffekten führt bei gegebenen Bildungsangeboten sowie institutionalisierten Sortier- und Selektionsleistungen des stratifizierten und segmentierten Bildungssystems zu sozial selektiven „Überlebenschancen" bis hin zum Erwerb der Hochschulreife und damit zur sozialen Selektivität von Studienberechtigten (Erikson u. Jonsson 1996; Blossfeld u. Shavit 1993). Es ist eine notwendige Voraussetzung für soziale Ungleichheiten von Studienchancen. Die darauf folgenden Bildungsentscheidungen von studienberechtigten Absolventen sind, abgesehen von institutionellen Voraussetzungen, die für die Aufnahme eines Hochschulstudiums erfüllt sein müssen, als notwendige wie hinreichende Bedingungen ursächlich für die soziale Ungleichheit beim Hochschulzugang.

Aus guten Gründen kommt dem sekundären Herkunftseffekt eine größere Bedeutung für die Bildungschancen von Abiturienten zu. Dies ist erstens deswegen der Fall, weil es sich auch bei den Abiturienten aus unteren Sozialschichten um eine selektive Gruppe leistungsstärkerer Schüler handelt, die zuvor alle Hürden im Bildungsverlauf überwunden haben (Blossfeld u. Shavit 1993). Im Unterschied zu den frühen Bildungsübergängen von der Grundschule in die Schullaufbahnen in der Sekundarstufe I dürften primäre Herkunftseffekte am Ende der Schulausbildung die anstehende, zwischen den Sozialschichten differierende Ausbildungsentscheidung nicht mehr ausreichend erklären (vgl. Breen u. Goldthorpe 1997). Im stratifizierten und segmentierten Bildungssystem Deutschlands wird zweitens wegen dem Ausscheiden leistungsschwächerer Schulkinder von Bildungsstufe zu Bildungsstufe der sekundäre Herkunftseffekt immer bedeutsamer für den Verbleib im Bildungssystem (Hillmert 2005, S. 177). Der im Zuge der Bildungsexpansion erfolgte Abbau von sozialer Selektivität in den unteren Stufen des Bildungswesens wird drittens durch eine zunehmende Selektivität in den höheren Stufen zumindest teilweise kompensiert (Becker 2006): „Je universeller Bildung im Primar- und Sekundarbereich wird, umso wahrscheinlicher ist es, dass Ungleichheit produzierende Mechanismen verstärkt im Tertiärbereich zum Zuge kommen“ (Müller u. Pollak 2008, S. 308). 
Deswegen müssten bildungspolitische Maßnahmen, die auf der einen Seite das Ziel haben, ungenutzte Bildungsreserven in den ,bildungsfernen“ Sozialschichten zu mobilisieren, und auf der anderen Seite das Ziel haben, soziale Ungleichheiten beim Hochschulzugang zu minimieren, vornehmlich am sekundären Herkunftseffekt, dem zwischen den Sozialschichten variierendem Bildungsverhalten, ansetzen. Ob diese Schlussfolgerung, die zwar für den Übergang auf die Sekundarstufe empirisch abgesichert ist (Stockè 2007; Erikson 2007; Becker 2000), auch für Studienchancen gerechtfertigt ist, ist jedoch im Folgenden empirisch zu klären. Weil aber die Effizienz und Effektivität solcher Programme erst nach längerer Zeit unter erheblichem Aufwand einer Evaluation und bei hohen Kosten für ganze Geburtsjahrgänge oder Generationen festgestellt werden können, wird im vorliegenden Beitrag in Anlehnung an die Arbeiten von Boudon (1974, 1979), Nash (2003) und Müller-Benedict (2007) mittels Simulationen mit realen Daten die Frage zu klären versucht, welche Wirkungen bestimmte Maßnahmen im Hinblick auf unsere Problemstellung haben werden. Welche bildungspolitischen Maßnahmen sind unter Qualitäts- und Kostengesichtspunkten tatsächlich geeignet, Talente aus bildungsfernen Gruppen für ein Hochschulstudium zu gewinnen? Diese Frage kann zudem spezifiziert werden, ob eine etwaige Reform des Schulsystems berücksichtigt werden soll: Was könnte man tun, wenn das Schulsystem, aus welchen Gründen auch immer, nicht verändert werden soll oder kann, und dennoch mehr Arbeiterkinder für ein Hochschulstudium gewonnen werden sollen? Und welche Resultate würde man für den Hochschulzugang erzielen, wenn bei einer Schulstrukturreform der frühe Übergang in die weiterführenden Schullaufbahnen der Sekundarstufe I fokussiert wird?

\section{Datenbasis und Variablen}

\subsection{Datenbasis}

Für die Analysen und Simulationen werden erstens Umfragedaten von sächsischen Abiturienten in den Abschlussklassen an allgemeinbildenden Gymnasien, beruflichen Gymnasien und Fachoberschulen aus den Jahren 2000, 2002, 2004 und 2006 herangezogen. Diese Daten wurden im Auftrag des Sächsischen Staatsministeriums für Kultur jeweils zu Anfang 2000, 2002, 2004 und 2006 in sächsischen Regionalschulbezirken (Bautzen, Chemnitz, Dresden, Leipzig und Zwickau) erhoben (Wolter et al. 2006). Die Erhebungen erfolgten an zufällig ausgewählten Schulen und umfassen jeweils 10 Prozent der Schüler in den Abschlussklassen an allgemein bildenden Gymnasien, beruflichen Gymnasien und Fachoberschulen. Für die einzelnen Erhebungszeitpunkte liegen schriftlich mit einem standardisierten Fragebogen erhobene Informationen für jeweils rund 2000 Personen in der 12. bzw. 13. Klassenstufe vor. Im Folgenden werden nur Befragte berücksichtigt, die sich sicher waren, was sie unmittelbar nach dem Abitur tun werden. Unberücksichtigt bleiben unentschlossene Befragte, weil für diese Personen der sekundäre Herkunftseffekt nicht berechnet werden kann. Von den ursprünglichen 7175 Befragten verbleiben dann und bei Kontrolle der für unsere Problemstellung auswertbaren Informationen mehr als 5300 Befragte in der Analysestichprobe. Weil es keine Selektivitäten der Unentschlossenheit nach sozialer Herkunft der Studienberechtigten gibt, auch nicht unter den Arbei- 
terkindern, dürften sich keine stichprobenbedingten Verzerrungen für die Simulationen ergeben (vgl. Becker u. Hecken 2007, S. 114, 2008, S. 27).

Für den Übergang von Gymnasiasten in der Sekundarstufe I in die Sekundarstufe II (Berufsausbildung vs. gymnasiale Oberstufe) werden zweitens Daten von PISA-E 2000 (die nationale Stichprobenergänzung der internationalen, von der OECD durchgeführten Hauptstudie von PISA 2000) verwendet (Baumert u. Artelt 2003). Während in der Hauptstudie rund 180.000 Jugendliche im Alter von 15 Jahren in 32 OECD-Ländern und Partnerstaaten erfasst wurden, umfasst das deutsche Sample rund 5000 deutsche Schüler in 219 Schulen. Bei PISA-E wurde die Zahl der Schulen auf 1460 erhöht. Die Stichprobenergänzung beruht auf zwei sich überlappenden Stichproben von 33809 15-Jährigen und 33766 Neuntklässlern. Von diesen 45899 Schülern werden in den folgenden Analysen nur die deutschen Gymnasiasten in der neunten Schulklasse berücksichtigt. Bei Berücksichtigung gültiger Werte für die soziale Herkunft, die Schulleistungen und die Bildungsentscheidung steht eine Analysestichprobe mit 6467 Fällen zur Verfügung.

Und schließlich werden drittens für den Übergang von der Primar- auf die Sekundarstufe I Umfragedaten des Konstanzer Panels über „Bildungsverläufe in Arbeiterfamilien“" herangezogen, das von Fauser (1984) durchgeführt wurde. Berücksichtigt wird die zweite Befragung von deutschen Eltern mit Kindern in verschiedenen Bundesländern (Baden-Württemberg, Nordrhein-Westfalen, Niedersachsen und West-Berlin), die im Herbst 1983 nach der Bildungsentscheidung und dem erfolgten Übergang in die weiterführenden Schulen der Sekundarstufe I erfolgte. An der ersten Befragung nahmen 4252 Eltern und an der zweiten Befragung noch 3085 Eltern teil. Für 2505 Schulkinder liegen nach Ausschluss fehlender Werte für die Simulation auswertbare Informationen vor. Nach Ansicht von Fauser (1984, S. 13) unterscheiden sich die beiden Befragungen in zentralen Ergebnissen kaum voneinander. Ausfälle gab es vor allem bei Arbeiterfamilien, weniger gebildeten Familien und Familien mit niedrigeren Schulwünschen für ihre Kinder. Eltern, die ihr Kind auf die Hauptschule schickten, sind gemessen an den tatsächlichen Übergangsquoten unterrepräsentiert. Weil Informationen zu den Ausfällen nicht verfügbar sind, ist es weder möglich, daraus resultierende Verzerrungen der Simulationsergebnisse abschätzen noch eine eventuell vorliegende Stichprobenverzerrung zu korrigieren (Becker 2000). Auf den ersten Blick scheinen diese Daten zu historisch für die vorliegende Problemstellung zu sein. Betrachtet man jedoch die Struktureffekte für den Übergang in das Gymnasium, so haben sich seit den 1980er Jahren so geringfügige Änderungen zwischen den Sozialschichten ergeben, dass diese Datengrundlage für aussagekräftige Simulationen herangezogen werden kann (Becker 2006). Zusätzliche Überprüfungen mit den historisch jüngeren Daten von IGLU-E 2001 (vgl. Becker u. Schubert 2006) bestätigen diese Vermutungen, sodass von reliablen Ergebnissen ausgegangen werden kann.

\subsection{Abhängige und unabhängige Variablen}

Für die Stichprobe der Abiturienten ist die Entscheidung von Studienberechtigten, im Anschluss an das Abitur an einer Universität oder Fachhochschule zu studieren oder eine andere Berufsausbildung (Berufsakademie oder betriebliche Lehre) zu beginnen, die abhängige Variable. In den weiterführenden Simulationen wird zwischen Hochschul- 
studium und nichttertiärer Berufsausbildung unterschieden. Die HIS-Studienberechtigten-Panelstudien belegen eine hohe Übereinstimmung von erfragter Ausbildungs- und Berufsentscheidung und dem tatsächlich realisierten Übergang. Heine u. Willich (2006, S. 4-5) zeigen hierbei Unterschiede zwischen den Abiturienten, die sich für eine Berufsausbildung entschieden haben, und denjenigen auf, die beabsichtigten zu studieren. Rund 88 Prozent der Studienberechtigten, die ein Studium planten, hatten ein Jahr später mit dem Studium begonnen oder beabsichtigen weiterhin, ein Studium zu beginnen. Die restlichen 12 Prozent dieser Personen haben ihre ursprüngliche Studienentscheidung revidiert oder sind sich nicht mehr sicher, ob sie ihre Entscheidung in die Tat umsetzen sollen. Mit 72 Prozent hat ein geringerer Anteil derjenigen Studienberechtigten, die sich zunächst für eine Berufsausbildung entschieden hatten, ihre Entscheidung realisiert. Rund ein Viertel derjenigen, die sich für eine Berufsausbildung entschieden hatten, hat bis zum nächsten Befragungszeitpunkt, d.h. ein Jahr später, die frühere Entscheidung revidiert und dann studiert (Heine u. Willich 2006, S. 4).

Für die Gymnasiasten in der 9. Klassenstufe ist der (beabsichtigte) Übergang in die gymnasiale Oberstufe die abhängige Variable. Zwar wurden die Schüler gefragt, was die höchste Ausbildung sein soll, die sie abschließen wollen. Aber der Wunsch, an einer Fachhochschule, einer Universität, Technischen Hochschule oder an einer anderen Hochschule (z.B. für Musik, Gestaltung) zu studieren, setzt für Gymnasiasten den Übergang in die gymnasiale Oberstufe und den anschließenden Erwerb der Fachhochschul- bzw. Hochschulreife voraus. Abgesehen von geringen Fehlerwahrscheinlichkeiten dürfte für Gymnasiasten diese Proxy-Variable ein valider Indikator für ihre Bildungsentscheidung sein.

Für die Schulkinder in der vierten bzw. sechsten Klassenstufe ist der Übergang von der Primar- auf die Sekundarstufe I die abhängige Variable. Hierbei wird zwischen Hauptschule, Realschule und Gymnasium unterschieden. Wegen geringer Fallzahlen bleiben Übergänge in die Sonder- und Gesamtschule unberücksichtigt (vgl. Becker 2000). Zudem wird als eine weitere abhängige Variable der elterliche Schulabschlusswunsch für ihre Kinder nach deren Bildungsübergang berücksichtigt, wobei zwischen Hochschulreife und anderen Schulabschlüssen unterschieden wird.

Die soziale Herkunft wird anhand der beruflichen Stellung des Haushaltsvorstands im Elternhaus gemessen. Der Übersichtlichkeit wegen wird zwischen den drei Sozialschichten, Arbeiter-, Mittel- und oberer Mittelschicht, unterschieden. Die Arbeiterschicht umfasst un- und angelernte Arbeiter, Facharbeiter, Vorarbeiter und Meister. Die Mittelschicht umfasst Angestellte mit einfacher (z.B. Verkaufsberufe) oder mit qualifizierter Tätigkeit (z. B. Sachbearbeiter) sowie Beamte im einfachen oder mittleren Dienst sowie Selbständige. Die obere Mittelschicht schließt hochqualifizierte Angestellte mit Leitungsfunktion (z.B. Prokurist), Angestellte mit Führungsaufgaben (z.B. Geschäftsführer), Beamte im gehobenen oder höheren Dienst (bzw. Richter) und akademische oder freie Berufe (z. B. Arzt mit eigener Praxis, Rechtsanwalt) ein. Diese Kategorisierung korrespondiert mit den theoretisch erwarteten schichtspezifischen Disparitäten für die Erträge, Kosten und Erfolgschancen, die von den Abiturienten in Bezug auf die Studienentscheidung erwartet werden. Dasselbe trifft für die Grundschulkinder mit den Schichtdisparitäten ihrer, anhand des Notendurchschnitts für Mathematik und Deutsch gemessenen, schulischen Leistung zu. Ebenfalls variieren die Schulleistungen der Gymnasiasten in der 
9. Klassenstufe (gemessen als gerundeter arithmetischer Mittelwert für die Zensuren in erster Fremdsprache, Mathematik, Deutsch, Biologie, Chemie und Physik) in erwarteter Weise zwischen den Sozialschichten (Tab. 1).

In der Einschätzung des Bildungsertrags B gibt es keine Schichtunterschiede (vgl. Becker u. Hecken 2008). Beim gewichteten Bildungsertrag pB, bei der die subjektiv erwartete Chance, diesen Bildungsertrag aufgrund der eigenen Erfolgserwartung realisieren zu können, berücksichtigt wird, liegen signifikante Unterschiede zwischen den Absolventen aus der oberen Mittelschicht einerseits und den anderen Sozialschichten andererseits vor (Somers' $d=-0,07 ; p \leq 0,001$ ). Des Weiteren spielt die subjektiv erwartete Erfolgswahrscheinlichkeit eine besondere Rolle für diese Bildungsentscheidung. Die Unterschiede in den individuell eingeschätzten Erfolgschancen zwischen den Sozialschichten sind zwar nicht sehr groß, aber statistisch signifikant (Somers' $d=0,09 ; p \leq 0,001$ ). Der Einfluss subjektiv erwarteter Kosten für ein Hochschulstudium wird anhand folgender Frage gemessen: „Haben die Kosten des Studiums Einfluss auf Ihre Entscheidung für oder gegen ein Studium?“ Die Antworten reichen von 1 für ,keinen Einfluss“ bis 5 für „sehr großen Einfluss“. Abgesehen davon, dass unklar ist, in welcher Richtung die Kosten die Studienentscheidung beeinflussen, könnte man zudem bei dieser Operationalisierung argumentieren, dass nicht die erwarteten Kosten gemessen werden, sondern retrospektiv die Auswirkung von Kosten auf die Entscheidung. Allerdings korreliert dieser Faktor (Somers' $d=-0,135 ; \mathrm{p} \leq 0,001$ ) in der theoretisch erwarteten Richtung und signifikant mit der sozialen Herkunft, so dass diese Variable verwendet werden kann, um die subjektiven Kostenerwartungen der Abiturienten abzubilden.

Tab. 1: Mittelwerte (in Klammern: Standardabweichung)

\section{Arbeiterschicht Mittelschicht Obere}

Mittelschicht

Nur Abiturienten $(\mathrm{n}=5361)$

Gewichteter Bildungsertrag $p B$

$3,90 \quad 3,92 \quad 4,25$

$(1=$ sehr niedrig $-6=$ sehr hoch $)$

Erfolgserwartung $p$

( $1=$ niedrig $-2=$ hoch $)$

1,56

$(0,50)$

$(0,49)$

Kostendruck $C$

3,13

2,88

( $1=$ sehr niedrig $-5=$ sehr hoch $)$

Nur Gymnasiasten in der 9. Klasse $(\mathrm{n}=6307)$

Durchschnittliche Schulnoten ( $1=$ sehr gut $-5=$ ungenügend $)$

Nur Primarschulkinder $(\mathrm{n}=2505)$

Durchschnittliche Schulnoten

Quellen: „Abiturientenbefragung in Sachsen 2000-06“, „PISA-E 2001“ und „Bildungsverläufe in Arbeiterfamilien“ - eigene Berechnungen. 


\section{Empirische Analysen und Befunde}

Die empirischen Analysen erfolgen in drei Schritten. Erstens werden in Simulationen für studienberechtigte Abiturienten die Wirkungen bildungspolitischer Maßnahmen gemessen, die entstehen, wenn primäre und sekundäre Herkunftseffekte neutralisiert, aber Reformen des Schulsystems nicht berücksichtigt werden. Zweitens werden für den Übergang von der Primar- auf die Sekundarstufe I und den späteren Übergang auf die Sekundarstufe II die Auswirkungen von Maßnahmen abgeschätzt, die zum Ziel haben, die Übertritte von Arbeiterkindern in das Gymnasium und damit auch die Chance zu erhöhen, dass sie ihre Schulausbildung mit der Studienberechtigung abschließen und nicht bereits frühzeitig vom Weg zum Hochschulstudium abgelenkt werden. Drittens werden die Wirkungen des aufeinander abgestimmten Zusammenspiels von Maßnahmen simuliert, die zum einen die Übergänge auf die Sekundarstufe I und II und zum anderen den Bildungsübergang von Abiturienten beeinflussen.

\subsection{Wie bedeutsam sind primäre und sekundäre Herkunftseffekte?}

In einem ersten Schritt werden die relativen Studienchancen in Abhängigkeit von der sozialen Herkunft beschrieben (Tab. 2). ${ }^{6}$ Während fast drei Viertel der Absolventen aus der oberen Mittelschicht zu studieren beabsichtigen, entscheiden sich fast zwei Drittel der Jugendlichen aus der Mittelschicht und etwas mehr als die Hälfte der studienberechtigten Arbeiterkinder für ein Universitäts- oder Fachhochschulstudium. Die relative Chance, dass sozial privilegierte Abiturienten aus der oberen Mittelschicht ein Hochschulstudium beginnen, ist doppelt so hoch wie für Arbeiterkinder. Abiturienten aus der Arbeiterschicht hingegen haben eine doppelt so große Chance wie Absolventen aus der oberen Mittelschicht, sich für eine nichttertiäre Berufsausbildung zu entscheiden. Offensichtlich werden sie eher von den Hochschulen ,weggelenkt“" als Abiturienten aus mittleren und höheren Sozialschichten.

Das gerichtete Zusammenhangsmaß Somers' d mit einem Wert von 0,137 ist hochsignifikant. Damit es keinen statistisch signifikanten Zusammenhang zwischen Schichtzugehörigkeit und Ausbildungsentscheidung gibt, also die Entscheidung für ein Hochschulstudium oder nichttertiäre Berufsausbildung nicht mehr von der sozialen Herkunft abhängt, müssten, so die Summe der Abweichungen der erwarteten Häufigkeiten $E\left(h_{i j}\right)$ von den beobachteten absoluten Häufigkeiten $h_{i j}:|597,6-478|+|940,9-888|+\ldots+$ $|651,3-692|+|455,1-325|$, insgesamt 708 der 5361 Abiturienten (13 Prozent) ihre Ausbildungsentscheidung revidieren (Tab. 2). Diese Abweichungen erwarteter von den beobachteten Entscheidungen ergeben sich durch das Zusammenspiel primärer und sekundärer Herkunftseffekte für diejenigen, die bis zum Abitur im Schulsystem verblieben sind (vgl. Boudon u. Bourricaud 1992, S. 622-624). Sie beschreiben somit das gesamte Ausmaß

6 Einen weiteren Hinweis für die Zuverlässigkeit der hier herangezogenen Daten ist ihre Entsprechung mit dem tatsächlichen Übergang ins Hochschulstudium: Im Jahre 2005 erwarben in Sachsen rund 37 Prozent der 18- bis 20-Jährigen die Hochschulreife und von diesen begannen rund 68 Prozent ein Hochschulstudium (Statistisches Landesamt Sachsen 2006). Unseren Daten zufolge entscheiden sich rund 62 Prozent für ein Hochschulstudium. 
Tab. 2: Ausbildungsentscheidung von Abiturienten nach sozialer Herkunft

\begin{tabular}{lcccc}
\hline & Arbeiterschicht & Mittelschicht & Obere Mittelschicht & Gesamt \\
\hline Universität & $32,8 \%$ & $38,7 \%$ & $51,7 \%$ & $41,0 \%$ \\
$\mathrm{~h}_{1 .}$ & 478 & 888 & 830 & 2196 \\
$\mathbf{E}\left(\mathbf{h}_{\mathbf{1}}\right)$ & $\mathbf{5 9 7 , 6}$ & $\mathbf{9 4 0 , 9}$ & $\mathbf{6 5 7 , 4}$ & \\
Fachhochschule & $22,5 \%$ & $21,9 \%$ & $18,4 \%$ & $21,0 \%$ \\
$\mathrm{~h}_{2 .}$ & 328 & 504 & 295 & 1127 \\
$\mathbf{E}\left(\mathbf{h}_{\mathbf{2}}\right)$ & $\mathbf{3 0 6 , 7}$ & $\mathbf{4 8 2 , 9}$ & $\mathbf{3 3 7 , 4}$ & \\
Berufsakademie & $10,3 \%$ & $9,3 \%$ & $9,7 \%$ & $9,7 \%$ \\
$\mathrm{~h}_{3 .}$ & 150 & 213 & 155 & 518 \\
$\mathbf{E}\left(\mathbf{h}_{\mathbf{3}}\right)$ & $\mathbf{1 4 1 , 0}$ & $\mathbf{2 2 1 , 9}$ & $\mathbf{1 5 5 , 1}$ & \\
Berufsausbildung & $34,5 \%$ & $30,1 \%$ & $20,2 \%$ & $28,4 \%$ \\
$\mathrm{~h}_{4 .}$ & 503 & 692 & 325 & 1520 \\
$\mathbf{E}\left(\mathbf{h}_{\mathbf{4}}\right)$ & $\mathbf{4 1 3 , 7}$ & $\mathbf{6 5 1 , 3}$ & $\mathbf{4 5 5 , 1}$ & \\
Gesamt & $100,0 \%$ & $100,0 \%$ & $100,0 \%$ & $100,0 \%$ \\
$\mathrm{~N}$ & 1459 & 2297 & 1605 & 5361 \\
\hline
\end{tabular}

Quelle: Abiturientenbefragung in Sachsen 2000-06 - eigene Berechnungen.

herkunftsbedingter Ungleichheit von Ausbildungsentscheidungen studienberechtigter Absolventen.

Da sich der Hochschulzugang aus dem Zusammenspiel primärer und sekundärer Herkunftseffekte ergibt, wäre es gemäß der hier herangezogenen Theorie notwendig, das selbst eingeschätzte individuelle Leistungspotenzial als intervenierende Variable zwischen sozialer Herkunft und Hochschulzugang zu berücksichtigen. Aufgrund der sozialen Selektivität der Übergänge an den einzelnen Gelenkstellen sowie der leistungsbezogenen Sortier- und Selektionsleistung des deutschen Schulsystems ,überlebt“ bis zum Ende der Schulzeit eine selektive Gruppe leistungsstarker Schüler. Weil die Wahl für ein Hochschulstudium oder für eine andere Ausbildung in der Regel eine individuelle Entscheidung von Abiturienten ist, die nur in wenigen Fällen durch Fremdselektion (etwa über Abiturnoten bei Numerus Clausus) bestimmt wird, erfolgt eine Selbstselektion eher über die subjektive Erwartung des entscheidenden Individuums, ein Hochschulstudium oder eine andere Ausbildung erfolgreich bewältigen und dadurch auch subjektiv erwartete Bildungserträge realisieren zu können. Da diese Erfolgserwartung hochgradig mit der vorherigen Schulperformanz korreliert und systematisch nach sozialer Herkunft variiert, bildet sie das individuelle Leistungspotenzial im Sinne primärer Herkunftseffekte ab (vgl. Becker u. Hecken 2009). Um Komplexität zu reduzieren wird zwischen hoher und niedriger Erfolgserwartung für ein Hochschulstudium unterschieden.

Während rund 67 Prozent der Absolventen aus der oberen Mittelschicht und 60 Prozent der Abiturienten aus der Mittelschicht eine hohe Erwartung aufweisen, ein Hochschulstudium erfolgreich bewältigen zu können, verfügen rund 56 Prozent der studienberechtigten Arbeiterkinder über eine hohe Erfolgserwartung (Tab.3). Offensichtlich führen die einzelnen Übergänge von der Primarstufe bis in die gymnasiale Oberstufe zur sukzessiven Homogenisierung der Gymnasiasten nach schulischer Performanz und Erfolgserwar- 
Tab. 3: Erfolgserwartung nach sozialer Herkunft

\begin{tabular}{lcccr}
\hline & Arbeiterschicht & Mittelschicht & Obere Mittelschicht & Gesamt \\
\hline Hoch & $55,7 \%$ & $59,5 \%$ & $66,7 \%$ & $60,6 \%$ \\
Niedrig & $44,3 \%$ & $40,5 \%$ & $33,3 \%$ & $39,4 \%$ \\
Gesamt & $100,0 \%$ & $100,0 \%$ & $100,0 \%$ & $100,0 \%$ \\
\hline
\end{tabular}

Quelle: Abiturientenbefragung in Sachsen 2000-06 - eigene Berechnungen.

tung, sodass leistungsbezogene Abstände zwischen den Sozialschichten nicht sehr groß erscheinen (Solga u. Wagner 2007).

In einem weiteren Schritt betrachten wir, um primäre Herkunftseffekte quantifizieren zu können, den Zusammenhang zwischen Erfolgserwartung und Bildungsentscheidung. Dieser wird, fokussierend auf die Entscheidung für ein Hochschulstudium im Vergleich zur Berufsausbildung, separat für die Sozialschichten betrachtet (Tab.4). Während sich rund 78 Prozent der vom Studienerfolg überzeugten Absolventen aus der oberen Mittelschicht für ein Hochschulstudium entscheiden, ist dies für jeweils 71 Prozent der Schüler aus der Mittel- oder Arbeiterschicht der Fall. Auch bei den Absolventen mit niedrigen oder unsicheren Erfolgserwartungen neigen eher die Jugendlichen aus der höheren Sozialschicht (mehr als die Hälfte) zu einem Hochschulstudium als die studienberechtigten Arbeiterkinder (knapp mehr als ein Drittel). Dass von den Absolventen mit derselben Erfolgserwartung ein deutlich geringer Anteil aus der Arbeiterschicht auf die Hochschule geht als aus der oberen Mittelschicht, offenbart den sekundären Effekt der sozialen Herkunft.

Der gerichtete Zusammenhang zwischen Erfolgserwartung und Bildungsentscheidung ist für alle Sozialschichten statistisch signifikant und in der Größenordnung (Somers' d

Tab. 4: Entscheidung für Hochschulstudium nach Erfolgserwartung und sozialer Herkunft

\begin{tabular}{|c|c|c|c|c|}
\hline \multirow{2}{*}{$\begin{array}{l}\text { Soziale } \\
\text { Herkunft }\end{array}$} & \multirow[t]{2}{*}{ Bildungsentscheidung } & \multicolumn{2}{|c|}{ Erfolgserwartung } & \multirow[t]{2}{*}{ Gesam } \\
\hline & & Niedrig & Hoch & \\
\hline \multirow[t]{5}{*}{ Obere Mittelschicht } & Kein Studium & $45,0 \%$ & $22,3 \%$ & $29,9 \%$ \\
\hline & Universität & $38,9 \%$ & $58,1 \%$ & $51,7 \%$ \\
\hline & Fachhochschule & $16,1 \%$ & $19,5 \%$ & $18,4 \%$ \\
\hline & Gesamt & $100,0 \%$ & $100,0 \%$ & $100,0 \%$ \\
\hline & Kein Studium & $55,1 \%$ & $28,7 \%$ & $39,4 \%$ \\
\hline \multirow[t]{4}{*}{ Mittelschicht } & Universität & $25,8 \%$ & $47,4 \%$ & $38,7 \%$ \\
\hline & Fachhochschule & $19,1 \%$ & $23,8 \%$ & $21,9 \%$ \\
\hline & Gesamt & $100,0 \%$ & $100,0 \%$ & $100,0 \%$ \\
\hline & Kein Studium & $64,6 \%$ & $29,0 \%$ & $44,8 \%$ \\
\hline \multirow[t]{3}{*}{ Arbeiterschicht } & Universität & $19,8 \%$ & $43,1 \%$ & $32,8 \%$ \\
\hline & Fachhochschule & $15,6 \%$ & $27,9 \%$ & $22,5 \%$ \\
\hline & Gesamt & $100,0 \%$ & $100,0 \%$ & $100,0 \%$ \\
\hline
\end{tabular}

Quelle: Abiturientenbefragung in Sachsen 2000-06 - eigene Berechnungen. 
zwischen 0,2 und 0,3) ähnlich. Würde man davon ausgehen, dass es in Abhängigkeit von der sozialen Herkunft keinen statistisch signifikanten Zusammenhang zwischen Erfolgserwartung und Ausbildungsentscheidung gibt, dann müssten 259 der 5361 Abiturienten ihre Ausbildungsentscheidung revidieren (vgl. Tab. A1 im Anhang). Diese Anzahl von 256 Revisionen entspricht einem prozentualen Anteil von rund 37 Prozent an allen 708 Revisionen, die notwendig wären, damit Chancengleichheit nach sozialer Herkunft bestünde. Anders ausgedrückt: Die tatsächlich bestehende Ungleichheit wird zu 37 Prozent durch primäre Herkunftseffekte und, komplementär dazu, zu 63 Prozent durch sekundäre Herkunftseffekte hervorgerufen (vgl. Erikson 2007; Jackson et al. 2007). ${ }^{7}$ Damit ist (wiederum) empirisch gesichert, dass sekundäre Herkunftseffekte für die Beschreibung und Erklärung sozial selektiver Zugänge zu Hochschulen weitaus bedeutsamer sind als primäre Herkunftseffekte.

\subsection{Welche bildungspolitische Maßnahme scheint geeignet zu sein?}

Auch wenn der sekundäre Herkunftseffekt zunächst deutlich größer als der primäre Herkunftseffekt erscheint, ist immer noch nicht gesichert, ob Maßnahmen, die als geeignet angesehen werden, sekundäre Herkunftseffekte zu reduzieren, auch tatsächlich zur Steigerung der Studienanfängerquote oder zur Verringerung der sozialen Ungleichheit von Studienchancen beitragen. Daher wird nun mittels realer Daten simuliert, wie sich das Ausschalten primärer und sekundärer Herkunftseffekte auf den Hochschulzugang auswirkt.

Hierbei wird anlog zu Boudon (1974) und Müller-Benedict (2007) vorgegangen und zunächst die Tab. 3 und 4 reorganisiert, in dem die Erfolgserwartungen mit dem Übergang auf die Hochschulen kombiniert werden (Tab.5). Weil die mittlere und höhere Sozialschicht die Referenzkategorien für die Absolventen aus der Arbeiterschicht sind, fassen wir, auch nicht zuletzt, um Komplexität zu reduzieren, die beiden höheren Sozialschichten zu einer Kategorie zusammen.

In einem ersten Schritt wird der primäre Effekt sozialer Herkunft „,neutralisiert“, indem für die Arbeiterkinder die gleichen Erfolgserwartungen angenommen werden wie für die Absolventen aus der Mittel- und Oberschicht. Diese Verteilung der subjektiv erwarteten Erfolgschancen (siehe Tab. 3) wird in Beziehung zu den Übergangswahrscheinlichkeiten für die Arbeiterkinder gesetzt (siehe Tab.4). Läge für die studienberechtigten Arbeiterkinder die Verteilung der Erfolgserwartungen wie für die Absolventen aus den höheren Sozialschichten vor, dann würden sich:

7 Im Unterschied zu Nash (2003) und Müller-Benedict (2007) wird hier eine gänzlich andere Berechnung der Herkunftseffekte verwendet, die auch eher der Definition von Herkunftseffekten nach Boudon (1974) entspricht. Weil der Herkunftseffekt die Unterschiede von Bildungserfolgen zwischen Sozialschichten meint, d.h. die Variation von Bildungsübergängen infolge der schichtspezifischen Leistungspotenziale und Bildungsentscheidungen, kann es keine separaten Herkunftseffekte für die einzelnen Sozialschichten geben. In der Terminologie der Regressionsanalyse wären primäre und sekundäre Herkunftseffekte die Varianz der Bildungserfolge, die durch die Herkunftsvariablen statistisch ,erklärt“ wird. Herkunftseffekte können daher entweder nur insgesamt für alle Sozialschichten oder im relativen Vergleich zwischen den Sozialschichten berechnet und interpretiert werden. 
Tab. 5: Erfolgserwartung und Entscheidung für Studium an der Universität oder Fachhochschule

\begin{tabular}{lll}
\hline Aus der & \multicolumn{2}{l}{ Mittel- und oberen Mittelschicht } \\
\cline { 2 - 3 } & Niedrig & Hoch \\
\hline haben Erfolgserwartung & $37,5 \%$ & $62,5 \%$ \\
und gehen auf die Hochschule & $48,6 \%$ & $74,1 \%$ \\
\hline Aus der & Arbeiterschicht & \\
\cline { 2 - 3 } & Niedrig & Hoch \\
\hline haben Erfolgserwartung & $44,3 \%$ & $55,7 \%$ \\
und gehen auf die Hochschule & $35,4 \%$ & $71,0 \%$ \\
\hline
\end{tabular}

Quelle: Abiturientenbefragung in Sachsen 2000-06 - eigene Berechnungen.

\section{7,5 Prozent $\cdot 0,354+62,5$ Prozent $\cdot 0,71=57,7$ Prozent}

dieser Arbeiterkinder für ein Hochschulstudium entscheiden. Das sind rund 2,5 Prozentpunkte mehr als die faktische Entscheidung von 55,2 Prozent der studienberechtigten Arbeiterkinder für ein Hochschulstudium.

Kommen wir zum zweiten Schritt: Wie groß ist die Steigerung der Studienanfängerquote bei den Abiturienten aus der Arbeiterschicht, wenn sie bei Berücksichtigung der unterschiedlichen Erfolgserwartungen (und in diesem Sinne bei Kontrolle der primären Herkunftseffekte) die gleichen Übergangswahrscheinlichkeiten wie die Absolventen aus den anderen Sozialschichten aufweisen würden? Neutralisiert man den sekundären Effekt der sozialen Herkunft, dann entscheiden sich:

\section{4,3 Prozent $\cdot 0,486+55,7$ Prozent $\cdot 0,741=62,8$ Prozent}

dieser Arbeiterkinder für ein Hochschulstudium. Dies bedeutet eine Steigerung der Übergänge um rund 8 Prozentpunkte. Statt der 806 Arbeiterkinder mit Hochschulreife würden nunmehr 914 Arbeiterkinder an der Universität studieren. Wenn der sekundäre Herkunftseffekt neutralisiert wird, dann steigert sich die Zahl der an die Universität wechselnden Arbeiterkinder um rund 13 Prozent.

Quantitativ bemessen wären in Bezug auf die Ausschöpfung von Bildungsreserven in „bildungsfernen“ Gruppen auf die Neutralisierung sekundärer Herkunftseffekte abzielende Maßnahmen wirksamer als die Neutralisierung primärer Herkunftseffekte. Und die herkunftsbedingte, den Prämissen der Chancengerechtigkeit widersprechende Ungleichheit zwischen diesen Sozialschichten in Bezug auf die hier betrachtete Bildungsbeteiligung würde zurückgehen. Hierbei sollte man die Einflüsse primärer Herkunftseffekte auf die Ausbildungsentscheidung von Abiturienten berücksichtigen (vgl. Becker u. Hecken 2009).

\subsection{Was würde die Kompensation erwarteter Studienkosten erbringen?}

Wie müsste eine bildungspolitische Maßnahme konkret aussehen, um diese beiden Ziele, Steigerung der Studienanfängerquote bei gleichzeitiger Senkung der sozialen Ungleichheit des Hochschulzugangs, zu erreichen? Ein zentrales Ergebnis von Becker u. Hecken 
Tab. 6: Einfluss der unmittelbaren Studienkosten auf die Bildungsentscheidung

\begin{tabular}{llllll}
\hline Aus der & \multicolumn{4}{l}{ Mittel- und oberen Mittelschicht } \\
\cline { 2 - 6 } & Sehr gering & Gering & Mittel & Hoch & Sehr hoch \\
\hline haben Kostendruck & $22,0 \%$ & $22,6 \%$ & $22,7 \%$ & $21,8 \%$ & $10,9 \%$ \\
und gehen auf die Hochschule & $70,9 \%$ & $75,9 \%$ & $67,4 \%$ & $54,2 \%$ & $42,6 \%$ \\
\hline Aus der & Arbeiterschicht & & & & \\
\cline { 2 - 6 } & Sehr gering & Gering & Mittel & Hoch & Sehr hoch \\
\hline haben Kostendruck & $14,9 \%$ & $17,5 \%$ & $25,3 \%$ & $25,8 \%$ & $16,8 \%$ \\
und gehen auf die Hochschule & $54,9 \%$ & $67,1 \%$ & $58,8 \%$ & $54,4 \%$ & $39,2 \%$ \\
\hline
\end{tabular}

Quelle: Abiturientenbefragung in Sachsen 2000-06 - eigene Berechnungen.

(2007, 2008) war, dass der ökonomische Kostendruck ein einflussreicher Faktor ist, der entscheidend zu den sekundären Herkunftseffekten zu Lasten der Arbeiterkinder unter den Abiturienten beiträgt, während sich die Abiturienten unterschiedlicher sozialer Herkunft bei den subjektiv erwarteten Renditen nicht signifikant voneinander unterscheiden (siehe Tab. 1).

Während rund 43 Prozent der Abiturienten aus der Arbeiterschicht die unmittelbar auftretenden Studienkosten als hoch oder sehr hoch einschätzen, teilt fast ein Drittel der Abiturienten aus den höheren Sozialschichten diese Einschätzung (Tab. 6). Rund ein Drittel der studienberechtigten Arbeiterkinder stuft im Unterschied zu den Abiturienten aus der Mittel- oder Oberschicht (25 Prozent) die erwarteten Studienkosten als (sehr) gering ein.

Welches Ergebnis würde man erzielen, wenn die unmittelbar erwarteten Kosten für ein Hochschulstudium neutralisiert werden? In diesem Falle entscheiden sich: 22,0 Prozent $\cdot 0,549+22,6$ Prozent $\cdot 0,671+22,7$ Prozent $\cdot 0,588+21,8$ Prozent $\cdot 0,544+10,9$ Prozent $\cdot 0,392=56,7$ Prozent der studienberechtigten Arbeiterkinder dafür, zu studieren. Man würde eine Steigerung des Universitätszugangs von „bildungsfernen“ Gruppen von anderthalb Prozentpunkten erzielen. Gemessen an der Wirkung, die durch eine unspezifische Neutralisierung des gesamten sekundären Herkunftseffekts erzielt wird, kommt der gezielten Neutralisierung des unmittelbaren Kostendrucks nach Erwerb der Hochschulreife zwar eine geringe, aber für die Steuerung des Hochschulzugangs nicht zu vernachlässigende Bedeutung zu (vgl. Jackson et al. 2007). ${ }^{8}$

Denn mit unseren Daten werden die gesamten Kosteneffekte unterschätzt, da „bildungsferne“ Gruppen nicht zuletzt aufgrund der vergleichsweise größeren sozialen Dis-

8 Nur für eine Minderheit der leistungsstarken Abiturienten aus den Arbeiterschichten scheint die Finanzierung eines Studiums kein Problem darzustellen, während die Mehrheit aufgrund des bescheidenen Einkommens und Vermögens ihrer Eltern auf eine staatliche Förderung angewiesen ist (Durrer u. Heine 2000). Der 18. Sozialerhebung des Deutschen Studentenwerks über die wirtschaftliche und soziale Lage der Studierenden in Deutschland zufolge werden die meisten Studierenden durch ihre Eltern unterstützt, die auch die Hauptlast der Studienkosten für ihre Kinder tragen. In der Zeit von 1991 bis 2006 sind die finanziellen Leistungen der Eltern für die Deckung der Studienkosten ihrer Kinder auf 52 Prozent gestiegen, während der Anteil des BAföG im gleichen Zeitraum von 20 Prozent auf 14 Prozent gesunken ist. Die reale Erhöhung der finanziellen Leistungen der Eltern liegt bei 19 Prozent, während die Leistungen nach dem BAföG um 28 Prozent abgenommen haben (Isserstedt et al. 2007, S. 16-22). 
tanz zur weiterführenden Schulbildung und akademischen Ausbildung sowie der damit verbundenen höheren Kosten und kürzeren Zeithorizonte bereits vor dem Übergang in das Gymnasium oder in die Gymnasiale Oberstufe alternative Bildungswege gewählt haben, die nicht zu den Hochschulen führen. Der Hintergrund dieser Überlegung ist, dass Akademikerfamilien oder Familien mit einem Hochschulabschluss aufgrund der geringen sozialen Distanz zur tertiären Ausbildung nicht nur geringere kurzfristige Teilnahmekosten, sondern auch langfristig deutlich geringere Informations-, Opportunitätsund Transaktionskosten haben müssten. Allerdings kann diese Annahme aufgrund der begrenzten Datengrundlage nicht überprüft und muss der zukünftigen Forschung überlassen werden.

\subsection{Muss die Bedeutung von Bildungserträgen nahegebracht werden?}

Welche Rolle spielen die subjektiv erwarteten und an den Arbeitsmarktchancen von Akademikern gemessenen Erträge eines Hochschulstudiums? Betrachtet man die jeweiligen Verteilungen der erwarteten Bildungserträge, so bestehen geringfügige Unterschiede zwischen den Sozialschichten (Tab.7). Demzufolge müssten die Arbeiterkinder nicht von den privilegierten Arbeitsmarktchancen der Hochschulabsolventen überzeugt werden. Würde man sie bei gegebenen Erwartungen von Bildungserträgen und subjektiv erwarteten Chancen, diese Erträge realisieren zu können, zu einem Hochschulstudium motivieren, dann würden 57,1 Prozent der studienberechtigten Arbeiterkinder und damit rund 2 Prozentpunkte mehr Arbeiterkinder als de facto studieren.

Gezielte Informationskampagnen, in denen die Vorteile der Hochschulausbildung offengelegt und zugleich verdeutlicht wird, wie leicht sie auch für studienbegabte Absolventen aus den „bildungsfernen“ Gruppen zu realisieren sind, bringen nur eine geringe zusätzliche Steigerung des Hochschulzugangs. Vielmehr müssten Einflüsse kompensiert werden, welche die „bildungsfernen“ Gruppen vom Hochschulstudium ablenken, auch wenn sie von den Bildungserträgen der tertiären Bildung überzeugt sind. Auch dieses Faktum unterstreicht die Bedeutung sekundärer Herkunftseffekte bei der Ausbildungsentscheidung von studienberechtigten Schulabgängern.

Tab. 7: Einfluss der erwarteten Bildungserträge auf die Bildungsentscheidung

\begin{tabular}{|c|c|c|c|c|c|}
\hline \multirow[t]{2}{*}{ Aus der } & \multicolumn{5}{|c|}{ Mittel- und oberen Mittelschicht } \\
\hline & Sehr gering & Gering & Mittel & Hoch & Sehr hoch \\
\hline $\begin{array}{l}\text { erwarten Bildungserträge } \\
\text { und gehen auf die } \\
\text { Hochschule }\end{array}$ & $\begin{array}{r}8,2 \% \\
31,8 \%\end{array}$ & $\begin{array}{l}17,1 \% \\
52,6 \%\end{array}$ & $\begin{array}{l}20,6 \% \\
56,8 \%\end{array}$ & $\begin{array}{l}11,6 \% \\
66,9 \%\end{array}$ & $\begin{array}{l}42,5 \% \\
78,9 \%\end{array}$ \\
\hline \multirow[t]{2}{*}{ Aus der } & \multicolumn{5}{|c|}{ Arbeiterschicht } \\
\hline & Sehr gering & Gering & Mittel & Hoch & Sehr hoch \\
\hline $\begin{array}{l}\text { erwarten Bildungserträge } \\
\text { und gehen auf die } \\
\text { Hochschule }\end{array}$ & $\begin{array}{l}10,3 \% \\
24,2 \%\end{array}$ & $\begin{array}{l}16,4 \% \\
42,9 \%\end{array}$ & $\begin{array}{l}24,4 \% \\
42,1 \%\end{array}$ & $\begin{array}{l}9,7 \% \\
61,0 \%\end{array}$ & $\begin{array}{l}39,2 \% \\
75,4 \%\end{array}$ \\
\hline
\end{tabular}

Quelle: Abiturientenbefragung in Sachsen 2000-06 - eigene Berechnungen. 
Tab. 8: Elterliche Bildungsentscheidung am Ende der Grundschulzeit nach sozialer Herkunft

\begin{tabular}{llccc}
\hline & Arbeiterschicht & Mittelschicht & Obere Mittelschicht & Gesamt \\
\hline Hauptschule & $31,1 \%$ & $10,7 \%$ & $6,3 \%$ & $15,3 \%$ \\
$\mathrm{~h}_{1 .}$ & 226 & 112 & 46 & 384 \\
$\mathbf{E}\left(\mathbf{h}_{\mathbf{1}}\right)$ & $\mathbf{1 1 1 , 4}$ & $\mathbf{1 6 0 , 7}$ & $\mathbf{1 1 1 , 9}$ & \\
Realschule & $41,5 \%$ & $34,8 \%$ & $18,4 \%$ & $32,0 \%$ \\
$\mathrm{~h}_{2 .}$ & 302 & 365 & 134 & 801 \\
$\mathbf{E}\left(\mathbf{h}_{\mathbf{2}}\right)$ & $\mathbf{2 3 2 , 5}$ & $\mathbf{3 3 5 , 1}$ & $\mathbf{2 3 3 , 4}$ & \\
Gymnasium & $27,4 \%$ & $54,5 \%$ & $75,3 \%$ & $52,7 \%$ \\
$\mathrm{~h}_{3 .}$ & 199 & 571 & 550 & 1320 \\
$\mathbf{E}\left(\mathbf{h}_{\mathbf{3}}\right)$ & $\mathbf{3 8 3 , 1}$ & $\mathbf{5 5 2 , 2}$ & $\mathbf{3 8 4 , 7}$ & \\
Gesamt & $100,0 \%$ & $100,0 \%$ & $100,0 \%$ & $100,0 \%$ \\
& 727 & 1048 & 730 & 2505 \\
\hline
\end{tabular}

Quelle: „Bildungsverläufe in Arbeiterfamilien“ - eigene Berechnungen.

\subsection{Würde eine Schulstrukturreform zum gewünschten Erfolg beitragen?}

Bislang wurde auf die Wirkung von Maßnahmen zur Neutralisierung primärer und sekundärer Herkunftseffekte für die sozial und leistungsbezogen selektive Gruppe von Abiturienten eingegangen, ohne dass die institutionelle Steuerung des Hochschulzugangs berücksichtigt wurde. Nunmehr werden für den Übergang von der Primar- auf die Sekundarstufe I die Auswirkungen von Maßnahmen abgeschätzt, die zum Ziel haben, die frühzeitige Ablenkung von Arbeiterkindern vom Weg zum Hochschulstudium auszuschalten. Damit soll die Frage beantwortet werden, ob eine Schulstrukturreform, wie etwa Aufhebung früher Bildungsübergänge nach der vierten bzw. sechsten Klassenstufe, zu höheren Übergangsraten von Arbeiterkindern auf das Gymnasium als eine der Voraussetzungen für den späteren Erwerb der Hochschulberechtigung führen würde. Eine andere Frage wäre, ob man eher in die Verbesserung der Schulleistungen und damit der Erfolgswahrscheinlichkeiten von Arbeiterkindern investieren sollte, um sie, ohne dass sie zuvor auf dem Weg zum Abitur scheitern, an die Hochschulen zu bringen.

Während drei Viertel der Kinder aus der oberen Mittelschicht und mehr als die Hälfte der Mittelschichtkinder nach der Grundschulzeit auf das Gymnasium wechseln, tut dies etwas mehr als ein Viertel der Arbeiterkinder (Tab. 8). Somit haben die Kinder aus der höheren Sozialschicht 8-mal und Mittelschichtkinder 3-mal bessere Chancen, auf das Gymnasium und damit auf den direkten Weg zur Hochschule zu gelangen als Arbeiterkinder (Somers' $d=0,33 ; p \leq 0,001$ ). Kinder aus höheren Sozialschichten haben bessere durchschnittliche Schulnoten, die für den Übergang in das Gymnasium vorteilhaft sind. Mehr als die Hälfte der Kinder aus der mittleren und höheren Sozialschicht, aber nur etwas mehr als ein Drittel der Arbeiterkinder weisen gute bis sehr gute Schulleistungen auf (Tab.9). Allerdings wechseln mit einem Anteil von 54 Prozent deutlich weniger leistungsstarke Arbeiterkinder auf das Gymnasium als Kinder aus höheren Sozialschichten (84 Prozent).

Da der Summe absoluter Abweichungen der erwarteten von den tatsächlichen Bildungsübergängen zufolge die Bildungsentscheidung für insgesamt 796 von 2505 Schul- 
Tab. 9: Schulleistung und elterliche Bildungsentscheidung am Ende der Grundschulzeit

\begin{tabular}{lllc}
\hline Aus der & \multicolumn{3}{l}{ Mittel- und oberen Mittelschicht } \\
\cline { 2 - 4 } & Sehr gut bzw. gut & Mittelmäßig & Schlecht \\
\hline haben Schulperformanz & $55,0 \%$ & $37,7 \%$ & $7,3 \%$ \\
und gehen auf das Gymnasium & $83,8 \%$ & $42,8 \%$ & $10,9 \%$ \\
\hline Aus der & Arbeiterschicht & & \\
\cline { 2 - 4 } & Sehr gut bzw. gut & Mittelmäßig & Schlecht \\
\hline haben Schulperformanz & $35,9 \%$ & $48,1 \%$ & $16,0 \%$ \\
und gehen auf das Gymnasium & $54,0 \%$ & $16,3 \%$ & $0,9 \%$ \\
\hline
\end{tabular}

Quelle: „Bildungsverläufe in Arbeiterfamilien“- eigene Berechnungen.

kindern revidiert werden müsste, um den Zusammenhang des Bildungsübergangs mit der sozialen Herkunft aufzulösen, und 173 Schulkinder bei Kontrolle ihrer Schulleistung in eine andere Schullaufbahn platziert werden müssten (vgl. Tab. A2 im Anhang), beträgt der Anteil der primären Herkunftseffekte an der gesamten Ungleichheit von Bildungschancen rund 22 Prozent und derjenige von sekundären Herkunftseffekten 78 Prozent.

Würde man den primären Effekt der sozialen Herkunft neutralisieren, dann würden etwa 36 Prozent der Arbeiterkinder auf das Gymnasium wechseln, was eine Steigerung um rund 9 Prozentpunkte bedeuten würde. Bei einer Neutralisierung der sekundären Herkunftseffekte würden bei einer Steigerung um 25 Prozentpunkte genau 52,4 Prozent der Arbeiterkinder ihre Schulausbildung auf dem Gymnasium fortsetzen. Oder anders ausgedrückt: Bei Kontrolle ihrer Schulleistung würden dann doppelt so viele Arbeiterkinder das Gymnasium besuchen als dies tatsächlich der Fall ist. ${ }^{9}$ Allerdings bedeutet der hier vorliegende Befund nicht, dass primäre Herkunftseffekte neutralisierende Maßnahmen generell zu vernachlässigen wären. Es sind beispielsweise von Büchel et al. (1997) oder von Becker u. Lauterbach (2008) relativ starke Bildungseffekte vorschulischer Betreuung nachgewiesen worden, die auf die Abschwächung sozialer Ungleichheit von Lernvoraussetzungen bei der Einschulung abzielten, und positive Bildungserfolge für Kinder aus unteren Sozialschichten erbrachten.

\subsection{Was würde eine erhöhte Durchlässigkeit des Schulsystems bringen?}

Von der OECD (2007) wird auch die geringe Durchlässigkeit des gegliederten deutschen Schulsystems kritisiert, die dazu beiträgt, dass „bildungsferne“ Gruppen vom Erwerb der Hochschulreife und damit vom Zugang zur tertiären Ausbildung ,abgelenkt“ werden (Müller u. Pollak 2008). Schulartwechsel, Nachholen von Schulabschlüssen oder

9 Dass Ditton u. Krüsken (2006) für den Bildungsübergang in Bayern zum Ergebnis kommen, dass der primäre den sekundären Herkunftseffekt dominiert, liegt wohl daran, dass in Bayern die Bildungsempfehlung verbindlich ist. Im von uns verwendeten Datensatz überwiegen die Bundesländer, in denen der Elternwille Vorrang hat. So müsste man systematisch untersuchen, $\mathrm{ob}$ in den Bundesländern mit verbindlicher Grundschulempfehlung primäre Herkunftseffekte die Bildungschancen dominieren, während in den anderen Bundesländern sekundäre Herkunftseffekte die Bildungsübergange strukturieren. 
die Aufbesserung schulischer Abschlüsse im Anschluss an den ersten Abschluss einer allgemeinbildenden Schule wie etwa Erwerb der Fach- oder Hochschulreife auf beruflichen Schulen wären institutionelle Maßnahmen, „bildungsferne“ Gruppen unabhängig von ihrer Selbstplatzierung in die vom Hochschulstudium ablenkenden Schullaufbahnen für den Erwerb der Studienberechtigung und für das Hochschulstudium zu gewinnen (vgl. Schuchart u. Maaz 2007; Schuchart 2006). Korrekturen ursprünglicher Bildungsentscheidungen in Richtung einer höheren Bildung über Schulartwechsel oder nachholenden Schulabschluss (Zweiter Bildungsweg) werden wiederum vornehmlich von privilegierten Schichten vorgenommen, die bereits beim Übergang von der Primar- auf die Sekundarstufe I höhere Bildungsmotivationen und Beteiligungschancen aufweisen (Henz 1997a, b). Die ,zweite Chance“ trägt eher zur sozialen Selektivität von Bildungschancen und somit kaum zum Abbau sozialer Ungleichheit von Studienchancen bei (Hillmert u. Jacob 2004).

Mit den hier herangezogenen Daten können wir diese Forschungsergebnisse replizieren. Weil keine Informationen über tatsächliche Korrekturen der ersten Bildungsentscheidung verfügbar sind, verwenden wir behelfsmäßig den elterlichen Schulabschlusswunsch. Dieser Indikator ist den Befunden von Schuchart u. Maaz (2007) zufolge eine valide Annäherung an das tatsächliche Bildungsverhalten. So wünschen sich 16 Prozent der Eltern in mittleren und höheren Sozialschichten für ihr Kind die Fachhochschulreife oder das Abitur, obwohl es zum Befragungszeitpunkt die Hauptschule besucht (Tab. 10). Hingegen äußern nur 4 Prozent der Arbeitereltern diesen Bildungswunsch für ihr Kind in der Hauptschule. Die relative Chance, dass sozial privilegierte Eltern diese Bildungsvorstellung verfolgen, ist 4,6-mal größer als für die Arbeiterschicht. Mehr als ein Drittel der Eltern in der mittleren und höheren Sozialschicht streben für ihr Kind die Studienberechtigung an, auch wenn es die Realschule besucht, aber nur 18 Prozent der Arbeitereltern. Hierbei sind, gemessen an den Chancenverhältnissen, die Abstände zwischen den Sozialschichten nur halb so groß. Während fast alle Eltern in den mittleren und höheren Sozialschichten die Hochschulreife avisieren, während ihr Kind das Gymnasium besucht, verfolgen rund 85 Prozent der Arbeitereltern diesen Bildungswunsch.

Tab. 10: Konsequenzen einer Korrektur der ersten Bildungsentscheidung

\begin{tabular}{llll}
\hline Aus der & \multicolumn{3}{l}{ Mittel- und oberen Mittelschicht } \\
\cline { 2 - 4 } & Hauptschule & Realschule & Gymnasium \\
\hline besuchen folgende Schullaufbahn & $8,9 \%$ & $28,1 \%$ & $63,0 \%$ \\
und sollen Hochschulreife erlangen & $16,1 \%$ & $34,3 \%$ & $94,5 \%$ \\
\hline Aus der & Arbeiterschicht & & \\
\cline { 2 - 4 } & Hauptschule & Realschule & Gymnasium \\
\hline besuchen folgende Schullaufbahn & $31,1 \%$ & $41,5 \%$ & $27,4 \%$ \\
und sollen Hochschulreife erlangen & $4,1 \%$ & $17,9 \%$ & $84,8 \%$ \\
\hline Leistungsstarke Kinder aus der & Arbeiterschicht & & \\
& Hauptschule & Realschule & Gymnasium \\
\hline besuchen folgende Schullaufbahn & $6,5 \%$ & $39,5 \%$ & $54,0 \%$ \\
und sollen Hochschulreife erlangen & $5,9 \%$ & $14,9 \%$ & $85,7 \%$ \\
\hline
\end{tabular}

Quelle: „Bildungsverläufe in Arbeiterfamilien“ - eigene Berechnungen. 
Was aber erbringen innersystemische Durchlässigkeit und Zweiter Bildungsweg, wenn sich „,bildungsferne“ wie privilegierte Gruppen entscheiden? Neutralisiert man sekundäre Effekte der sozialen Herkunft, dann würden: 31,1 Prozent · 0,161 +41,5 Prozent $\cdot 0,343+27,4$ Prozent $\cdot 0,945=45,1$ Prozent der Eltern in der Arbeiterschicht für ihr Kind die Hochschulreife anstreben (Tab. 10). Würde man „bildungsfernen“ Gruppen die „Zweite Chance“ erleichtern, dann würden zusätzliche Möglichkeiten geschaffen, Arbeiterkinder für das Hochschulstudium zu gewinnen. Zumindest wären potenziell 45 Prozent statt 27 Prozent der Arbeiterkinder eines Jahrgangs auf dem Weg zum Hochschulstudium (vgl. Tab. 8). Sollten nur potenziell studienbegabte Arbeiterkinder diese zweite Chance erhalten sollen, dann hätten: 6,5 Prozent $\cdot 0,161+39,5$ Prozent $\cdot 0,343+54$ Prozent 0,945=65,6 Prozent (vgl. Tab. 10) statt 27 Prozent der Arbeiterkinder die Chance, die Hochschulreife zu erlangen und zu studieren. Auch diese Simulation belegt die besondere Bedeutung sekundärer Herkunftseffekte für die relativen Studienchancen von Arbeiterkindernn oder die soziale Ungleichheit von Studienchancen.

\subsection{Soll man den „Flaschenhals“ erweitern?}

Eingangs wurde darauf verwiesen, dass in Deutschland die gymnasiale Oberstufe der „Flaschenhals“ und der Erwerb der Hochschulberechtigung ein „Nadelöhr“ auf dem Weg zum Hochschulstudium ist. Der Übergang in die Sekundarstufe II ist wiederum ein Punkt, an dem Arbeiterkinder vom Weg zum Hochschulstudium abgelenkt werden (Becker u. Hecken 2008). Was würde es bringen, wenn, behielte man die soziale Selektivität beim Übergang von der Grundschule in die weiterführenden Schullaufbahnen der Sekundarstufe I und die geringe Durchlässigkeit des Schulsystems bei, man für Arbeiterkinder den Übergang in die gymnasiale Oberstufe erleichtern würde? Den Daten von PISA-E 2000 zufolge würden 74,2 Prozent der Arbeiterkinder im Gymnasium ihre Schulbildung auf der gymnasialen Oberstufe fortsetzen. Im Vergleich dazu beabsichtigen 87 Prozent der Gymnasiasten aus der mittleren und höheren Sozialschicht, auf die gymnasiale Oberstufe zu wechseln. Während sich die Gymnasiasten aus den unterschiedlichen Sozialschichten kaum in ihren Schulleistungen voneinander unterscheiden, so sind die Übergangsraten für die Gymnasiasten aus den höheren Sozialschichten in allen Leistungskategorien höher als für die Arbeiterkinder (Tab. 11).

Tab. 11: Schulleistung und Bildungsentscheidung gegen Ende der Sekundarstufe I

\begin{tabular}{llll}
\hline Aus der & \multicolumn{3}{l}{ Mittel- und oberen Mittelschicht } \\
\cline { 2 - 4 } & Sehr gut oder gut & Mittelmäßig & Schlecht \\
\hline haben Schulleistung & $33,8 \%$ & $51,0 \%$ & $15,2 \%$ \\
und gehen weiter auf das Gymnasium & $94,4 \%$ & $85,9 \%$ & $74,4 \%$ \\
\hline Aus der & Arbeiterschicht & & \\
\cline { 2 - 4 } & Sehr gut oder gut & Mittelmäßig & Schlecht \\
\hline haben Schulleistung & $29,9 \%$ & $53,7 \%$ & $16,5 \%$ \\
und gehen weiter auf das Gymnasium & $87,4 \%$ & $71,2 \%$ & $59,8 \%$ \\
\hline
\end{tabular}

Quelle: „PISA-E 2000“- eigene Berechnungen. 
Eine Neutralisierung primärer Herkunftseffekte würde eine Steigerung der Übergänge für die Arbeiterkinder um 0,7 Prozentpunkte erbringen und wäre daher weitgehend wirkungslos. Neutralisiert man die sekundären Herkunftseffekte, dann würden: 29,9 Prozent $\cdot 0,944+53,7$ Prozent $\cdot 0,859+16,5$ Prozent $\cdot 0,744=86,6$ Prozent der Arbeiterkinder in die gymnasiale Oberstufe wechseln. Die Neutralisierung sekundärer Herkunftseffekte erbringt eine Steigerung der Übergänge von Arbeiterkindern um 12,4 Prozentpunkte und würde eine deutliche Erweiterung des „Flaschenhalses“ bedeuten.

\subsection{Was würden aufeinander abgestimmte Maßnahmen erbringen?}

Einem nicht unerheblichen Reservoir von sicherlich studienbegabten Talenten aus ,bildungsfernen" Gruppen bleibt der Weg zu den Hochschulen vor allem wegen sekundärer Herkunftseffekte ,,versperrt“. Würde man für Arbeiterkinder die sekundären Herkunftseffekte am Ende der Grundschulzeit neutralisieren und sie für Gymnasiasten aus der Arbeiterschicht und studienberechtigte Arbeiterkinder unverändert lassen, dann würden $(0,524$ $\cdot 0,742 \cdot 0,552) \cdot 100$ Prozent $\approx 22 \operatorname{statt}(0,274 \cdot 0,742 \cdot 0,552) \cdot 100$ Prozent $\approx 11$ Prozent der Arbeiterkinder eines Geburtsjahrgangs an Hochschulen studieren. ${ }^{10}$ Würde man nur für studienberechtigte Arbeiterkinder die sekundären Herkunftseffekte reduzieren, dann würden $(0,274 \cdot 0,742 \cdot 0,628) \cdot 100$ Prozent $\approx 13$ statt 11 Prozent der Arbeiterkinder studieren. Würde man zudem die sekundären Herkunftseffekte beim Übergang in die gymnasiale Oberstufe reduzieren, dann würden $(0,274 \cdot 0,866 \cdot 0,628) \cdot 100$ Prozent $\approx 15$ statt 11 Prozent der Arbeiterkinder an die Hochschulen gelangen. Die Reduktion der Ablenkungswirkung infolge sekundärer Herkunftseffekte zu einem sehr frühen Zeitpunkt des Bildungsverlaufs würde eine wesentlich größere Wirkung erbringen als im Bildungsverlauf spät einsetzende Maßnahmen. Sie wäre auch erfolgversprechender, als wenn man nur auf die Neutralisierung primärer Herkunftseffekte setzen würde. Im letzteren Falle würden $(0,359 \cdot 0,749 \cdot 0,577) \cdot 100$ Prozent $\approx 16$ statt 11 Prozent der Arbeiterkinder studieren. Auch wenn beim ersten Bildungsübergang die primären und beim Übergang in die gymnasiale Oberstufe sowie nach dem Abitur die sekundären Herkunftseffekte neutralisiert würden, würden allenfalls 19 Prozent der Arbeiterkinder auf die Hochschulen gelangen.

Setzt man schließlich bei jedem der hier betrachteten Bildungsübergänge ausschließlich darauf, die sekundären Herkunftseffekte auszuschalten, dann würden, bei Kontrolle der Leistungspotenziale und Studienmotivationen, $(0,524 \cdot 0,866 \cdot 0,628) \cdot 100$ Prozent $\approx 28$ Prozent statt 11 Prozent der Arbeiterkinder eines Jahrgangs studieren. ${ }^{11}$ Setzt

10 Ohne neutralisierende Maßnahmen lassen sich die tatsächlichen Übergangsraten von 12 Prozent zu Anfang des 21. Jahrhunderts reproduzieren (vgl. Becker 2006): So würden unseren Daten zufolge eben $(0,274 \cdot 0,742 \cdot 0,552) \cdot 100$ Prozent $=11,2$ Prozent der Arbeiterkinder eines Jahrgangs studieren.

11 Wie bedeutsam sekundäre Herkunftseffekte sind, lässt sich am Zusammenspiel von Schullaufbahnempfehlung und tatsächlichem Bildungsverhalten ablesen (Tab. A3 im Anhang). Bei den hier betrachteten Bundesländern hat nur Baden-Württemberg eine für die Eltern weitgehend verbindliche Bildungsempfehlung, während die anderen Bundesländer den Eltern große Entscheidungsspielräume lassen. Während es in Bezug auf das Gymnasium in der Oberschicht eine Übereinstimmung der Bildungsempfehlung und des elterlichen Bildungsverhaltens gibt, 
man zudem die ,zweite Chance“ nach der ersten Bildungsentscheidung in Relation zur verbesserten Studienchance, dann würde man, vorausgesetzt, dass alle institutionellen Hürden bis zum Abitur wegfallen würden, ebenfalls $(0,451 \cdot 0,628) \cdot 100$ Prozent=28 Prozent der Arbeiterkinder für das Hochschulstudium gewinnen können.

Insgesamt zeigen die Simulationsergebnisse eindrücklich: Es müsste vornehmlich, mehr als ausschließlich auf die Leistungssteigerung der Kinder aus „bildungsfernen“ Gruppen, auf die frühzeitige Beeinflussung des Bildungsverhaltens gesetzt werden. Reformen der Schulstruktur und der Möglichkeiten, die Studienberechtigung zu erwerben, könnten die damals von Dahrendorf (1965a, b) und jüngst von der OECD geforderten Resultate erbringen.

\section{Zusammenfassung und Schlussfolgerung}

Ziel des vorliegenden Beitrags war es, eine theoretisch begründete und empirisch abgesicherte Antwort auf die Frage vorzulegen, wie „,bildungsferne“ Gruppen für ein Hochschulstudium gewonnen werden könnten. Diese Fragestellung ist zugleich ein empirische Anwendung der hier zugrunde gelegten Theorie, die zu erklären versucht, warum „bildungsferne" Gruppen vom Hochschulstudium fernbleiben (vgl. Becker u. Hecken 2007, 2008). Aus theoretischer Sicht wurde für das Fernbleiben der Arbeiterkinder von den Universitäten und Fachhochschulen zum einen das Zusammenspiel von primären Herkunftseffekten (den Wirkungen der Schichtzugehörigkeit auf erbrachte Leistungen und zukünftige Erfolgschancen) und sekundären Herkunftseffekten (den Wirkungen der sozialen Herkunft auf individuelle Entscheidungen über tertiäre Ausbildung) als empirisch abgesicherte Erklärung angeführt. Zum anderen wurde argumentiert, dass die Strukturen des allgemeinbildenden Schulsystems, die institutionellen Regelungen für die Allokation und das Verbleiben im Bildungssystem sowie das attraktive Bildungsangebot in der nichttertiären Berufsausbildung die Arbeiterkinder bei anstehenden Bildungsentscheidungen vom direkten Weg zur Hochschulausbildung ablenken.

In Bezug auf bildungs- oder hochschulpolitische Maßnahmen sollte empirisch geklärt werden, welchem Typus der Herkunftseffekte die größere Bedeutung und welche Rolle neben dem Elternhaus dem Bildungssystem zukommt. Daten über das Bildungsverhalten von sächsischen Abiturienten belegen, dass den Erwartungen, ein Hochschulstudium erfolgreich bewältigen zu können, eine vergleichsweise geringere Bedeutung für die suboptimale Ausschöpfung von Begabungsreserven in „bildungsfernen“ Gruppen zukommt. Vielmehr konnte empirisch belegt werden, dass sekundäre Herkunftseffekte dominieren, so dass motivierte wie studienbegabte Arbeiterkinder eher vom Zugang zu Hochschulen abgelenkt werden als Abiturienten aus höheren Sozialschichten. Aber hierbei liegt es nicht

schicken rund 30 Prozent der Arbeitereltern trotz der Gymnasialempfehlung ihre Kinder auf die Haupt- oder Realschule. Hingegen tendieren Elternhäuser in den höheren Sozialschichten bei einer Empfehlung für die Haupt- oder Realschule eher als die Arbeiterschicht dazu, ihre Kinder möglichst in eine höhere Schullaufbahn zu platzieren. Würden sich die Eltern in der Arbeiterschicht so verhalten wie die höheren Sozialschichten, dann würden 37 statt 27 Prozent ihrer Kinder auf das Gymnasium wechseln. 
daran, dass die Arbeiterschichten zu wenig von den vergleichsweise günstigen Bildungserträgen, die aus dem Hochschulstudium entstehen, überzeugt wären. Die „Bildungsferne" der Arbeiterkinder resultiert weder ausschließlich aus den im Vergleich zu den Kindern aus höheren Sozialschichten geringeren Leistungsmotivationen noch aus einer affektiven Abneigung gegen höhere Bildung, sondern aus Bildungsentscheidungen, die sich aus den Restriktionen und Opportunitäten der sozialen Herkunft sowie den Anreizen und Restriktionen des Bildungssystems ergeben. Die vorgelegten empirischen Befunde bestätigen wiederum eindeutig, dass sich die Abstinenz der Arbeiterkinder von den deutschen Hochschulen aus den Folgen kumulativer Bildungsentscheidungen und sozial selektiver Bildungsübergänge ergibt (Müller u. Karle 1993; Müller u. Pollak 2008).

Würde man primäre Herkunftseffekte neutralisieren, so könnte man den Übergang von studienberechtigten Arbeiterkindern in die Hochschulen um 5 Prozentpunkte steigern. Eine leistungsbezogene Auswahl von als studierfähig erachteten Abiturienten durch die Hochschulen würde nicht zwangsläufig zur weiteren Benachteiligung von Arbeiterkindern gegenüber den Abiturienten aus höheren Sozialschichten führen. Eine Neutralisierung sekundärer Herkunftseffekte hingegen erbringt eine deutliche Steigerung des Hochschulzugangs von 8 Prozentpunkten unter den Arbeiterkindern. Angenommen, die Rekrutierungspotenziale der höheren Sozialschichten und Akademiker wären weitgehend erschöpft, dann können in der Tat durch eine soziale Öffnung der Hochschulen bislang ungenutzte Begabungsreserven in der Arbeiterschicht mobilisiert werden. Dadurch könnte die Studienbeteiligung und die Akademikerquote in der Bevölkerung gesteigert werden.

Würde man zudem, so unsere Befunde, die frühe Selektion beim Übergang in die Schullaufbahnen der Sekundarstufe I und die spätere Selektion beim Übergang in die Sekundarstufe II ausschalten sowie die Möglichkeit, frühe Bildungsentscheidungen korrigieren zu können, verbessern, dann könnte die Ablenkung der Arbeiterkinder vom Weg zu den Hochschulen noch deutlicher reduziert werden. Pro Jahrgang würde sich die Studienbeteiligung der Arbeiterkinder mehr als verdoppeln. Auch zeigen die Simulationsergebnisse für junge Schulkinder am Ende der Grundschulzeit und für Gymnasiasten in der 9. Klassenstufe, dass die Neutralisierung sekundärer Herkunftseffekte effektiver wäre als Maßnahmen, um die Lernvoraussetzungen und Leistungspotenziale der Arbeiterkinder auf das Niveau der Schulkinder aus den höheren Sozialschichten zu bringen. So gesehen, stellt sich bei der sozialen Öffnung der Hochschulen die Schulstrukturfrage in Deutschland durchaus von Neuem. Denn auf die Reform des Hochschulzugangs abgestimmte Maßnahmen in den allgemeinbildenden Schulen dürften zusammen mit der Flexibilisierung des (nachholenden) Erwerbs einer Studienberechtigung und der größeren Durchlässigkeit der Sekundarstufen die größten Wirkungen für den Abbau der sozialen Ungleichheiten von Bildungschancen und die Steigerung der Studierquoten erbringen. Allerdings ist beim gegenwärtigen Kenntnisstand hervorzuheben, dass aufgrund unzureichender Datenlage die Neutralisierung einzelner Mechanismen (Kostendruck oder erwartete Bildungserträge) für den Übergang in die Sekundarstufe I oder II nicht abgeschätzt werden konnte. Dies müsste mit aktuellen wie informationsreichen Daten nachgeholt werden.

Da weder eine Reduktion sozioökonomischer Ungleichheiten unter den Elternhäusern (vgl. Erikson 1996) noch einschneidende Schulstrukturreformen derzeit wahrscheinlich sein dürften, stellt sich die Frage, welche hochschulpolitischen Maßnahmen nunmehr 
geeignet wären, um die Nachfrage der „,bildungsfernen“ Gruppen nach einem Hochschulstudium zu steigern und die ungerechtfertige soziale Schließung der Universitäten zu vermeiden (Mayer 2003)? Den bei einer unzureichenden Datengrundlage vorgelegten Befunden zufolge müsste sie weniger auf die Stärkung individueller Bildungsmotivationen unter den „,bildungsfernen Gruppen“ als auf die Verminderung von Investitionsrisiken den kurz- wie langfristigen Kostenerwartungen bei gegebenen Leistungspotentialen für die „,bildungsfernen“ Gruppen abzielen. Kostengünstiger und effektiver wäre es, die Möglichkeiten für nachholenden Erwerb einer Studienberechtigung zu erleichtern und die Durchlässigkeit beim Hochschulzugang zu erhöhen (Wolter 2009). 


\section{Anhang}

Tab. A1: Hochschulstudium nach Erfolgserwartung und sozialer Herkunft - Differenzen zwischen beobachteten und erwarteten Häufigkeiten

\begin{tabular}{|c|c|c|c|c|c|}
\hline \multirow[t]{2}{*}{ Bildungsentscheidung } & \multirow{2}{*}{\multicolumn{2}{|c|}{ Soziale Herkunft }} & \multicolumn{2}{|c|}{ Erfolgserwartung } & \multirow[t]{2}{*}{ Gesamt } \\
\hline & & & Hoch & Niedrig & \\
\hline \multirow{7}{*}{ Universität } & Oberschicht & $\mathrm{h}_{1 .}$ & 208 & 622 & 830 \\
\hline & & $\mathrm{E}\left(\mathrm{h}_{1}\right)$ & 217,7 & 612,3 & \\
\hline & Mittelschicht & $\mathrm{h}_{2}$ & 240 & 648 & 888 \\
\hline & & $\mathrm{E}\left(\mathrm{h}_{2}\right)$ & 232,9 & 655,1 & \\
\hline & Arbeiterschicht & $\mathrm{h}_{3}$ & 128 & 350 & 478 \\
\hline & & $\mathrm{E}\left(\mathrm{h}_{3}\right)$ & 125,4 & 352,6 & \\
\hline & Gesamt & & 576 & 1620 & 2196 \\
\hline \multirow{7}{*}{ Fachhochschule } & Oberschicht & $\mathrm{h}_{4 .}$ & 86 & 209 & 295 \\
\hline & & $\mathrm{E}\left(\mathrm{h}_{4}\right)$ & 95,5 & 199,5 & \\
\hline & Mittelschicht & $\mathrm{h}_{5}$ & 178 & 326 & 504 \\
\hline & & $\mathrm{E}\left(\mathrm{h}_{5}\right)$ & 163,2 & 340,8 & \\
\hline & Arbeiterschicht & $\mathrm{h}_{6 .}$ & 101 & 227 & 328 \\
\hline & & $\mathrm{E}\left(\mathrm{h}_{6}\right)$ & 106,2 & 221,8 & \\
\hline & Gesamt & & 365 & 762 & 1127 \\
\hline \multirow{7}{*}{ Berufsakademie } & Oberschicht & $\mathrm{h}_{7}$. & 55 & 100 & 155 \\
\hline & & $\mathrm{E}\left(\mathrm{h}_{7}\right)$ & 63,4 & 91,6 & \\
\hline & Mittelschicht & $\mathrm{h}_{8}$. & 89 & 124 & 213 \\
\hline & & $\mathrm{E}\left(\mathrm{h}_{8}\right)$ & 87,2 & 125,8 & \\
\hline & Arbeiterschicht & $h_{9}$ & 68 & 82 & 150 \\
\hline & & $\mathrm{E}\left(\mathrm{h}_{9}\right)$ & 61,4 & 88,6 & \\
\hline & Gesamt & & 212 & 306 & 518 \\
\hline \multirow{7}{*}{ Berufsausbildung } & Oberschicht & $\mathrm{h}_{10 .}$ & 186 & 139 & 325 \\
\hline & & $\mathrm{E}\left(\mathrm{h}_{10}\right)$ & 204,8 & 120,2 & \\
\hline & Mittelschicht & $\mathrm{h}_{11 .}$ & 423 & 269 & 692 \\
\hline & & $\mathrm{E}\left(\mathrm{h}_{11}\right)$ & 436,1 & 255,9 & \\
\hline & Arbeiterschicht & $\mathrm{h}_{12}$ & 349 & 154 & 503 \\
\hline & & $\mathrm{E}\left(\mathrm{h}_{12}\right)$ & 317,0 & 186,0 & \\
\hline & Gesamt & & 958 & 562 & 1520 \\
\hline
\end{tabular}

Quelle: Abiturientenbefragung in Sachsen 2000-06 - eigene Berechnungen. 
Tab. A2: Elterliche Bildungsentscheidung und Übergang in die Sekundarstufe I nach Schulleistung und sozialer Herkunft - Differenzen zwischen beobachteten und erwarteten Häufigkeiten

\begin{tabular}{|c|c|c|c|c|c|c|}
\hline \multirow[t]{2}{*}{ Bildungsentscheidung } & \multirow{2}{*}{\multicolumn{2}{|c|}{ Soziale Herkunft }} & \multicolumn{3}{|c|}{ Schulleistung } & \multirow[t]{2}{*}{ Gesamt } \\
\hline & & & Gut & Mittelmäßig & Schlecht & \\
\hline \multirow{7}{*}{ Hauptschule } & Oberschicht & $\mathrm{h}_{1 .}$ & 17 & 125 & 84 & 226 \\
\hline & & $\mathrm{E}\left(\mathrm{h}_{1}\right)$ & 18,2 & 114,8 & 93,0 & \\
\hline & Mittelschicht & $\mathrm{h}_{2}$ & 7 & 54 & 51 & 112 \\
\hline & & $\mathrm{E}\left(\mathrm{h}_{2}\right)$ & 9,0 & 56,9 & 46,1 & \\
\hline & Arbeiterschicht & & 7 & 16 & 23 & 46 \\
\hline & & $\mathrm{E}\left(\mathrm{h}_{3}\right)$ & 3,7 & 23,4 & 18,9 & \\
\hline & Gesamt & & 31 & 195 & 158 & 384 \\
\hline \multirow{6}{*}{ Realschule } & Oberschicht & $\mathrm{h}_{4}$ & 103 & 168 & 31 & 302 \\
\hline & & $\mathrm{E}\left(\mathrm{h}_{4}\right)$ & 93,1 & 181,7 & 27,1 & \\
\hline & Mittelschicht & $\mathrm{h}_{5}$. & 104 & 233 & 28 & 365 \\
\hline & & $\mathrm{E}\left(\mathrm{h}_{5}\right)$ & 112,6 & 219,6 & 32,8 & \\
\hline & Arbeiterschicht & $\mathrm{h}_{6}$ & 40 & 81 & 13 & 134 \\
\hline & & $\mathrm{E}\left(\mathrm{h}_{6}\right)$ & 41,3 & 80,6 & 12,0 & \\
\hline \multirow{8}{*}{ Gymnasium } & Gesamt & & 247 & 482 & 72 & 801 \\
\hline & Oberschicht & $\mathrm{h}_{7}$ & 141 & 57 & 1 & 199 \\
\hline & & $\mathrm{E}\left(\mathrm{h}_{7}\right)$ & 144,9 & 51,9 & 2,3 & \\
\hline & Mittelschicht & $\mathrm{h}_{8}$ & 402 & 159 & 10 & 571 \\
\hline & & $\mathrm{E}\left(\mathrm{h}_{8}\right)$ & 415,7 & 148,8 & 6,5 & \\
\hline & Arbeiterschicht & $h_{9}$ & 418 & 128 & 4 & 550 \\
\hline & & $\mathrm{E}\left(\mathrm{h}_{9}\right)$ & 400,4 & 143,3 & 6,2 & \\
\hline & Gesamt & & 961 & 344 & 15 & 1320 \\
\hline
\end{tabular}

Quelle: „Bildungsverläufe in Arbeiterfamilien“- eigene Berechnungen. 
Tab. A3: Grundschulempfehlung und elterliche Bildungsentscheidung nach sozialer Herkunft

\begin{tabular}{|c|c|c|c|c|c|}
\hline \multirow{2}{*}{$\begin{array}{l}\text { Soziale } \\
\text { Herkunft }\end{array}$} & \multirow{2}{*}{$\begin{array}{l}\text { Bildungs- } \\
\text { entscheidung }\end{array}$} & \multicolumn{3}{|c|}{ Grundschulempfehlung } & \multirow[t]{2}{*}{ Gesamt } \\
\hline & & Hauptschule & Realschule & Gymnasium & \\
\hline \multirow[t]{4}{*}{ Oberschicht } & Hauptschule & $63,8 \%$ & $8,1 \%$ & $1,0 \%$ & $7,4 \%$ \\
\hline & Realschule & $31,9 \%$ & $66,7 \%$ & $4,5 \%$ & $19,6 \%$ \\
\hline & Gymnasium & $4,3 \%$ & $25,2 \%$ & $94,5 \%$ & $72,9 \%$ \\
\hline & Gesamt & $100,0 \%$ & $100,0 \%$ & $100,0 \%$ & $100,0 \%$ \\
\hline \multirow[t]{4}{*}{ Mittelschicht } & Hauptschule & $66,9 \%$ & $6,6 \%$ & $0,7 \%$ & $11,8 \%$ \\
\hline & Realschule & $30,5 \%$ & $76,7 \%$ & $13,0 \%$ & $36,7 \%$ \\
\hline & Gymnasium & $2,5 \%$ & $16,7 \%$ & $86,3 \%$ & $51,6 \%$ \\
\hline & Gesamt & $100,0 \%$ & $100,0 \%$ & $100,0 \%$ & $100,0 \%$ \\
\hline \multirow[t]{4}{*}{ Arbeiterschicht } & Hauptschule & $84,9 \%$ & $15,6 \%$ & $2,6 \%$ & $33,2 \%$ \\
\hline & Realschule & $15,1 \%$ & $76,8 \%$ & $27,6 \%$ & $41,7 \%$ \\
\hline & Gymnasium & $0,0 \%$ & $7,6 \%$ & $69,9 \%$ & $25,2 \%$ \\
\hline & Gesamt & $100,0 \%$ & $100,0 \%$ & $100,0 \%$ & $100,0 \%$ \\
\hline
\end{tabular}

Quelle: „Bildungsverläufe in Arbeiterfamilien“- eigene Berechnungen.

\section{Literatur}

Baumert, Jürgen, und Cordula Artelt. 2003. Konzeption und technische Grundlagen der Studie. In Ein differenzierter Blick auf die Länder der Bundesrepublik Deutschland, Hrsg. Deutsches PISA-Konsortium PISA 2000, 11-50. Opladen: Leske + Budrich.

Becker, Rolf, und Anna Etta Hecken. 2007. Studium oder Berufsausbildung? Eine empirische Überprüfung der Modelle zur Erklärung von Bildungsentscheidungen von Esser sowie von Breen und Goldthorpe. Zeitschrift für Soziologie 36:100-117.

Becker, Rolf, und Anna Etta Hecken. 2008. Warum werden Arbeiterkinder vom Studium an Universitäten abgelenkt? Eine empirische Überprüfung der „Ablenkungsthese“ von Müller und Pollak (2007) und ihrer Erweiterung durch Hillmert und Jacob (2003). Kölner Zeitschrift für Soziologie und Sozialpsychologie 60:3-29.

Becker, Rolf, und Anna Etta Hecken. 2009. Higher education or vocational training? An empirical test of the rational action model of educational choices suggested by Breen and Goldthorpe (1997) and Esser (1999). Acta Sociologica 52:25-45.

Becker, Rolf, und Frank Schubert. 2006. Soziale Ungleichheit von Lesekompetenzen. Eine Matching-Analyse im Längsschnitt mit Querschnittsdaten von PIRLS 2001 und PISA 2000. Kölner Zeitschrift für Soziologie und Sozialpsychologie 58:253-284.

Becker, Rolf, und Wolfgang Lauterbach. 2008. Vom Nutzen vorschulischer Erziehung und Elementarbildung - Bessere Bildungschancen für Arbeiterkinder? In Bildung als Privileg, Hrsg. Rolf Becker, Wolfgang Lauterbach, 3. Aufl., 129-159. Wiesbaden: VS Verlag.

Becker, Rolf. 2000. Klassenlage und Bildungsentscheidungen. Eine empirische Anwendung der Wert-Erwartungstheorie. Kölner Zeitschrift für Soziologie und Sozialpsychologie 52:450-475.

Becker, Rolf. 2006. Dauerhafte Bildungsungleichheiten als unerwartete Folge der Bildungsexpansion? In Bildungsexpansion - Erwartete und unerwartete Folgen, Hrsg. Andreas Hadjar, Rolf Becker, 27-62. Wiesbaden: VS-Verlag für Sozialwissenschaften. 
Blossfeld, Hans-Peter, und Yossi Shavit. 1993. Dauerhafte Ungleichheiten. Zur Veränderung des Einflusses der sozialen Herkunft auf die Bildungschancen in dreizehn industrialisierten Ländern. Zeitschrift für Pädagogik 39:25-52.

Boudon, Raymond, und François Bourricaud. 1992. Soziologische Stichworte. Wiesbaden: Westdeutscher Verlag.

Boudon, Raymond. 1974. Education, opportunity, and social inequality. New York: Wiley.

Boudon, Raymond. 1979. Widersprüche sozialen Handelns. Darmstadt: Luchterhand.

Breen, Richard, und John H. Goldthorpe. 1997. Explaining educational differentials. Towards a formal rational action theory. Rationality and Society 9:275-305.

Büchel, Felix, C. Katharina Spieß, und Gert Wagner. 1997. Bildungseffekte vorschulischer Kinderbetreuung. Kölner Zeitschrift für Soziologie und Sozialpsychologie 49:528-539.

Dahrendorf, Ralf. 1965a. Arbeiterkinder an deutschen Universitäten. Tübingen: Mohr.

Dahrendorf, Ralf. 1965b. Bildung ist Bürgerrecht. Hamburg: Nannen.

Dahrendorf, Ralf. 1976. Bildung bleibt Bürgerrecht. Doch es heißt endgültig Abschied nehmen von gesellschaftspolitischen Illusionen. In Die Bildungsreform - eine Bilanz, Hrsg. Hellmut Becker, Ralf Dahrendorf, Peter Glotz, Hans Maier, 63-71. Stuttgart: Klett.

Ditton, Hartmut, und Jan Krüsken. 2006. Der Übergang von der Grundschule in die Sekundarstufe I. Zeitschrift für Erziehungswissenschaft 9:348-372.

Dobischat, Rolf. 2007. Statement des Präsidenten des Deutschen Studentenwerks auf der Pressekonferenz zur 18. Sozialerhebung des Deutschen Studentenwerks am 19. Juni 2007 in Berlin. http://www.studentenwerke.de/pdf/Statement_Dobischat18SE.pdf. (Stand: 15 Jan 2008).

Durrer, Franz, und Christoph Heine. 2000. Studienfinanzierung und Studierneigung im Freistaat Sachsen. Dresden: SMWK.

Erikson, Robert. 2007. Social selection in Stockholm schools: Primary and secondary effects on the transition to upper secondary education. In From origin to destination. Trends and mechanisms in social stratification research, Hrsg. Stefani Scherer, Reinhard Pollak, Gunnar Otte, Markus Gangl, 35-76. Frankfurt a. M.: Campus.

Erikson, Robert, und Jan O. Jonsson. 1996. Explaining class inequality in education: The swedish test case. In Can education be equalized?, Hrsg. Robert Erikson, Jan O. Jonsson, 1-63. Boulder: Westview Press.

Fauser, Richard. 1984. Der Übergang auf weiterführende Schulen. Soziale und schulische Bedingungen der Realisierung elterlicher Bildungserwartungen. Projekt: Bildungsverläufe in Arbeiterfamilien. Abschlußbericht 1. Konstanz: Universität Konstanz.

Goldthorpe, John H. 1996. Class analysis and the reorientation of class theory: The case of persisting differentials in educational attainment. British Journal of Sociology 47:481-501.

Heine, Christoph, und Julia Willich. 2006. Studienberechtigte 2005: Übergang in Studium, Ausbildung und Beruf. Hannover: HIS (Forum Hochschule 6/2006).

Henz, Ursula. 1997a. Der nachgeholte Erwerb allgemeinbildender Schulabschlüsse. Analysen zur quantitativen Entwicklung und sozialen Selektivität. Kölner Zeitschrift für Soziologie und Sozialpsychologie 49:223-241.

Henz, Ursula. 1997b. Der Beitrag von Schulformwechseln zur Offenheit des allgemeinbildenden Schulsystems. Zeitschrift für Soziologie 26:53-69.

Hillmert, Steffen, und Marita Jacob. 2003. Social inequality in higher education: Is vocational training a pathway leading to or away from university? European Sociological Review 19:319-334.

Hillmert, Steffen. 2005. Bildungsentscheidungen und Unsicherheit: soziologische Aspekte eines vielschichtigen Zusammenhangs. Zeitschrift für Erziehungswissenschaft 8:173-186.

Hillmert, Steffen, und Marita Jacob. 2004. Zweite Chance im Schulsystem. In Institutionalisierte Ungleichheiten? Stabilität und Wandel von Bildungschancen, Hrsg. Peter A. Berger, Heike Kahlert, 1-24. Weinheim: Juventa. 
Isserstedt, Wolfgang, Elke Middendorff, Gregor Fabian, und Andrä Wolter. 2007. Die wirtschaftliche und soziale Lage der Studierenden in der Bundesrepublik Deutschland 2006 - Ausgewählte Ergebnisse, Hrsg. vom Bundesministerium für Bildung und Forschung. Berlin: BMBF.

Jackson, Michelle, Robert Erikson, John Goldthorpe, und Meir Yaish. 2007. Primary and secondary effects in class differentials in educational attainment. Acta Sociologica 50:211-229.

Lüttinger, Paul. 1994. Studentenberge und Lehrlingstäler. Droht die Akademisierung der Gesellschaft? ISI (Informationsdienst Soziale Indikatoren) 2:1-3.

Mayer, Karl Ulrich. 2003. Das Hochschulwesen. In Das Bildungswesen in der Bundesrepublik Deutschland, Hrsg. Kai S. Cortina, Jürgen Baumert, Achim Leschinsky, Karl Ulrich Mayer, 581-624. Reinbek: Rowohlt.

Mayer, Karl Ulrich, Walter Müller, und Reinhard Pollak. 2007. Germany: Institutional change and inequalities of access in higher education. In Stratification in higher education, Hrsg. Yossi Shavit, Richard Arum, Adam Gamoran, 240-265. Stanford: Stanford University Press.

Müller, Walter, und Wolfgang Karle. 1993. Social selection in educational systems in Europe. European Journal of Sociology 9:1-23.

Müller, Walter, und Reinhard Pollak. 2008. Weshalb gibt es so wenige Arbeiterkinder in Deutschlands Universitäten? In Bildung als Privileg, Hrsg. Rolf Becker, Wolfgang Lauterbach, 3. Aufl., 307-346. Wiesbaden: VS Verlag.

Müller-Benedict, Volker. 2007. Wodurch kann die soziale Ungleichheit des Schulerfolgs am stärksten verringert werden? Kölner Zeitschrift für Soziologie und Sozialpsychologie 59:615-639.

Nash, Roy. 2003. Inequality/difference in education: Is a real explanation of primary and secondary effects possible? British Journal of Sociology 54:433-451.

OECD. 2007. Bildung auf einen Blick 2007 (Education At A Glance 2007). Briefing Notes für Deutschland (18.09.2007). www.oecd.org/dataoecd/22/28/39317467.pdf. (Stand: 11 Jan 2008).

Picht, Georg. 1964. Die deutsche Bildungskatastrophe. Olten: Walter.

Pross, Helge. 1969. Über die Bildungschancen von Mädchen in der Bundesrepublik. Frankfurt a. M.: Suhrkamp.

Schuchart, Claudia, und Kai Maaz. 2007. Schulbesuch und Elternaspiration am Ende der Sekundarstufe 1. Kölner Zeitschrift für Soziologie und Sozialpsychologie 59:640-666.

Schuchart, Claudia. 2006. Die Bedeutung der Entkopplung von Schulart und Schulabschluss für die Schullaufbahnplanung aus Elternsicht. Zeitschrift für Soziologie der Erziehung und Sozialisation 26:403-419.

Solga, Heike, und Sandra Wagner. 2007. Die Zurückgelassenen - die soziale Verarmung der Lernumwelt von Hauptschülerinnen und Hauptschülern. In Bildung als Privileg, Hrsg. Rolf Becker, Wolfgang Lauterbach, 187-215. Wiesbaden: VS Verlag für Sozialwissenschaften.

Statistisches Landesamt des Freistaates Sachsen. 2006. Studienberechtigte mit Fachhochschulreife nehmen zu, jedoch nur knapp 60 Prozent studieren. Pressemitteilung 62/2006 vom 24. März 2006. Kamenz URL http://www.statistik.sachsen.de/12/pressearchiv/archiv2006/pm06206. pdf.

Stockè, Volker. 2007. Explaining educational decision and effects of families' social class position: An empirical test of the Breen-Goldthorpe model of educational attainment. European Sociological Review 23:505-519.

Trautwein, Ulrich, Kai Maaz, Oliver Lüdtke, Gabriel Nagy, Nicole Husemann, Rainer Watermann, und Olaf Köller. 2006. Studieren an der Berufsakademie oder an der Universität, Fachhochschule oder Pädagogischen Hochschule? Ein Vergleich des Leistungsstands, familiären Hintergrunds, beruflicher Interessen und der Studienwahlmotive von (künftigen) Studierenden aus Baden-Württemberg. Zeitschrift für Erziehungswissenschaft 9:393-412. 
Wolter, Andrä. 2009. Soziale Differenzierungen der Zugangswege zur Hochschule im internationalen Vergleich. In Bildungsungleichheiten revisited, Hrsg. Heinz-Hermann Krüger, Ursula Rabe-Kleberg, Rolf-Torsten Kramer, Jürgen Budde. Wiesbaden: VS Verlag für Sozialwissenschaften.

Wolter, Andrä, Karl Lenz, und Rüdiger Laskowski. 2006. Studierbereitschaft bleibt auf hohem Niveau. Die Studien- und Berufswahl von Studienberechtigten des Abschlussjahrgangs 2006 in Sachsen. Eine empirische Untersuchung des Sächsischen Staatsministeriums für Kultus in Zusammenarbeit mit der Technischen Universität Dresden (Abschlussbericht). Dresden: SMK und TU Dresden.

Rolf Becker, 1960, Prof. Dr., Abteilung für Bildungssoziologie an der Universität Bern. Forschungsgebiete: Bildungssoziologie, Sozialstrukturanalyse, Lebensverlaufsforschung, Methoden der empirischen Sozialforschung und angewandte Statistik, Rational-Choice-Theorien, Arbeitsmarkt- und Mobilitätsforschung, empirische Wahlforschung. Veröffentlichungen (Auswahl): Lehrbuch der Bildungssoziologie. Wiesbaden 2009; Higher Education or Vocational Training? An Empirical Test of the Rational Action Model of Educational Choices Suggested by Breen and Goldthorpe (1997) and Esser (1999), Acta Sociologica 2009 (mit Anna Etta Hecken); Warum werden Arbeiterkinder vom Studium an Universitäten abgelenkt? Eine empirische Überprüfung der „Ablenkungsthese“ von Müller und Pollak (2007) und ihrer Erweiterung durch Hillmert und Jacob (2003), Kölner Zeitschrift für Soziologie und Sozialpsychologie 60, 2008 (mit Anna Etta Hecken). 